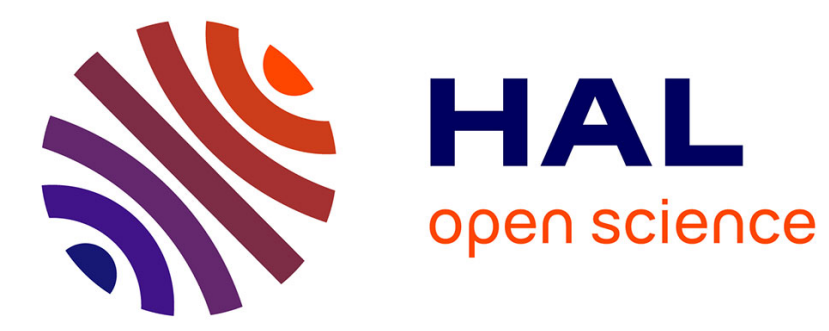

\title{
Point Source Regularization of the Finite Source Reflector Problem
}

Jean-David Benamou, Guillaume Chazareix, Giorgi Rukhaia, Wilbert L Ijzerman

\section{- To cite this version:}

Jean-David Benamou, Guillaume Chazareix, Giorgi Rukhaia, Wilbert L Ijzerman. Point Source Regularization of the Finite Source Reflector Problem. Journal of Computational Physics, 2022. hal$03344571 \mathrm{v} 2$

\section{HAL Id: hal-03344571 \\ https://hal.inria.fr/hal-03344571v2}

Submitted on 31 Jan 2022

HAL is a multi-disciplinary open access archive for the deposit and dissemination of scientific research documents, whether they are published or not. The documents may come from teaching and research institutions in France or abroad, or from public or private research centers.
L'archive ouverte pluridisciplinaire HAL, est destinée au dépôt et à la diffusion de documents scientifiques de niveau recherche, publiés ou non, émanant des établissements d'enseignement et de recherche français ou étrangers, des laboratoires publics ou privés. 


\section{Point Source Regularization of the Finite Source Reflector Problem}




\title{
Point Source Regularization of the Finite Source Reflector Problem
}

\author{
Jean-David Benamou ${ }^{\mathrm{a}}$, Guillaume Chazareix ${ }^{\mathrm{a}}$, Giorgi Rukhaia ${ }^{\mathrm{a}}$, Wilbert \\ IJzerman $^{\mathrm{c}, \mathrm{b}}$ \\ ${ }^{a}$ INRIA, 2, Rue Simone Iff, Paris, 75012, , France \\ ${ }^{b}$ Einhdhoven university of technology, De Zaale, Eindhoven, $5600 \mathrm{MB}$, , The Netherlands \\ ${ }^{c}$ SIGNIFY, High Tech Campus 48, Eindhoven, 5656 AE, , The Netherlands
}

\begin{abstract}
We address the "freeform optics" inverse problem of designing a reflector surface mapping a prescribed source distribution of light to a prescribed farfield distribution, for a finite light source. When the finite source reduces to a point source, the light source distribution has support only on the optics ray directions. In this setting, the inverse problem is well-posed for arbitrary source and target probability distributions. It can be recast as an Optimal Transportation problem and has been studied both mathematically and numerically. We are not aware of any similar mathematical formulation in the finite source case: i.e. the source has an "étendue" with support both in space and directions. We propose to leverage the well-posed variational formulation of the point source problem to build a smooth parameterization of the reflector and the reflection map. Under this parameterization, we can construct a smooth loss/misfit function to optimize for the best solution in this class of reflectors. Both steps, the parameterization and the loss, are related to Optimal Transportation distances. We also take advantage of recent progress in the numerical approximation and resolution of these mathematical objects to perform a numerical study.
\end{abstract}

Keywords: Inverse reflector problem; optimal transportation; non-linear optimization.

2000 MSC: 78A46, 49Q22, 65K10 


\section{Introduction}

\subsection{The optical setup}

The finite source/near field to far-field "freeForm reflector problem" is the inverse problem of determining the shape of a reflector surface mapping a given near field illuminance (a light source) to a desired far-field illumination. The light propagates in the simplest geometrical optics regime, the reflector is a perfect mirror. We describe here the mathematical modelization of two experimental setups: the point source to far-field target (figure 1) and the finite source to far-field target (figure 2).

\section{Modelization of the reflector}

The reflector is a surface $\mathcal{R}$ in $\mathbb{R}^{d}(d=2,3)$, it is parameterized using $x_{0} \in X_{0}:=\mathbb{S}_{+}^{d-1}$ the upper hemisphere and a given "radius" function $\rho \in$ $\mathcal{C}^{1}\left(X_{0}, \mathbb{R}\right)$. By convention, $x$ will denote an angular parameterization of $\mathbb{S}_{+}^{d-1}$ while $\hat{x}$ (with the $\hat{\text {.) }}$ will be the corresponding vector in $\mathbb{R}^{d}$ positioned at the origin. With these notations the reflector is modeled as:

$$
\mathcal{R}=\left\{\hat{x}_{0} \rho\left(x_{0}\right), x_{0} \in X_{0} \text { s.t. } \rho\left(\frac{\pi}{2}\right)=R\right\} .
$$

We constrain the reflector curve $\mathcal{R}$ to pass trough the point $(0, R)$ (in $\left.\mathbb{R}^{2}\right)$. This is easily generalized to $d=3, x$ is then a couple of (azimuth, elevation) angles.

We will keep to $d=2$ in the paper to simplify the presentation.

\section{Modelization of the illuminance}

The illuminance/light is a given probability distribution $\mu_{S}:\left(s, x_{s}\right) \in$ $S \times X_{s} \mapsto \mu_{s}\left(x_{s}\right) \in[0,1], \int_{S} \mu_{s}\left(X_{s}\right) d s=1$, where again $X_{s}=\mathbb{S}_{+}^{1}$ and $S$ is a "patch", subset of $\mathbb{R}^{d}$. It will be a subset of the horizontal axis containing the origin $0_{\mathbb{R}^{2}}$. In general we will always assume $S$ to be bounded and symmetric with respect to $0_{\mathbb{R}^{d}}$. This paper will simply consider $d=2$ and the segment

$$
S=[-\eta, \eta] \times\{0\}
$$

or its discretization.

In summary light rays leave the points $(s, 0) \in \mathbb{R}^{2}$ in the the direction $x_{s} \in X_{s} \subset \mathbb{S}_{+}^{1}$ with intensity/energy $\mu_{s}\left(x_{s}\right)$.

When $\eta \rightarrow 0$ or equivalently $R \rightarrow \infty$ we reach the point source illuminance regime, i.e $S \rightarrow\left\{0_{\mathbb{R}^{d}}\right\}$ and $\mu_{S}:=\mu_{0} \in \mathcal{P}\left(X_{0}\right)$ is a probability 
distribution in angles only. Note that the reflector shares its parameterization on $X_{0}$ with the illuminance $\mu_{0}$ at $s=0$.

\section{Modelization of the illumination}

The desired illumination is a given probability distribution $\nu: y \in Y \mapsto$ $\nu(y) \in[0,1], \nu(Y)=1$, where $Y:=\mathbb{S}_{-}^{d-1}$ the lower hemisphere angles.

\subsection{The Point Source Reflector Problem}

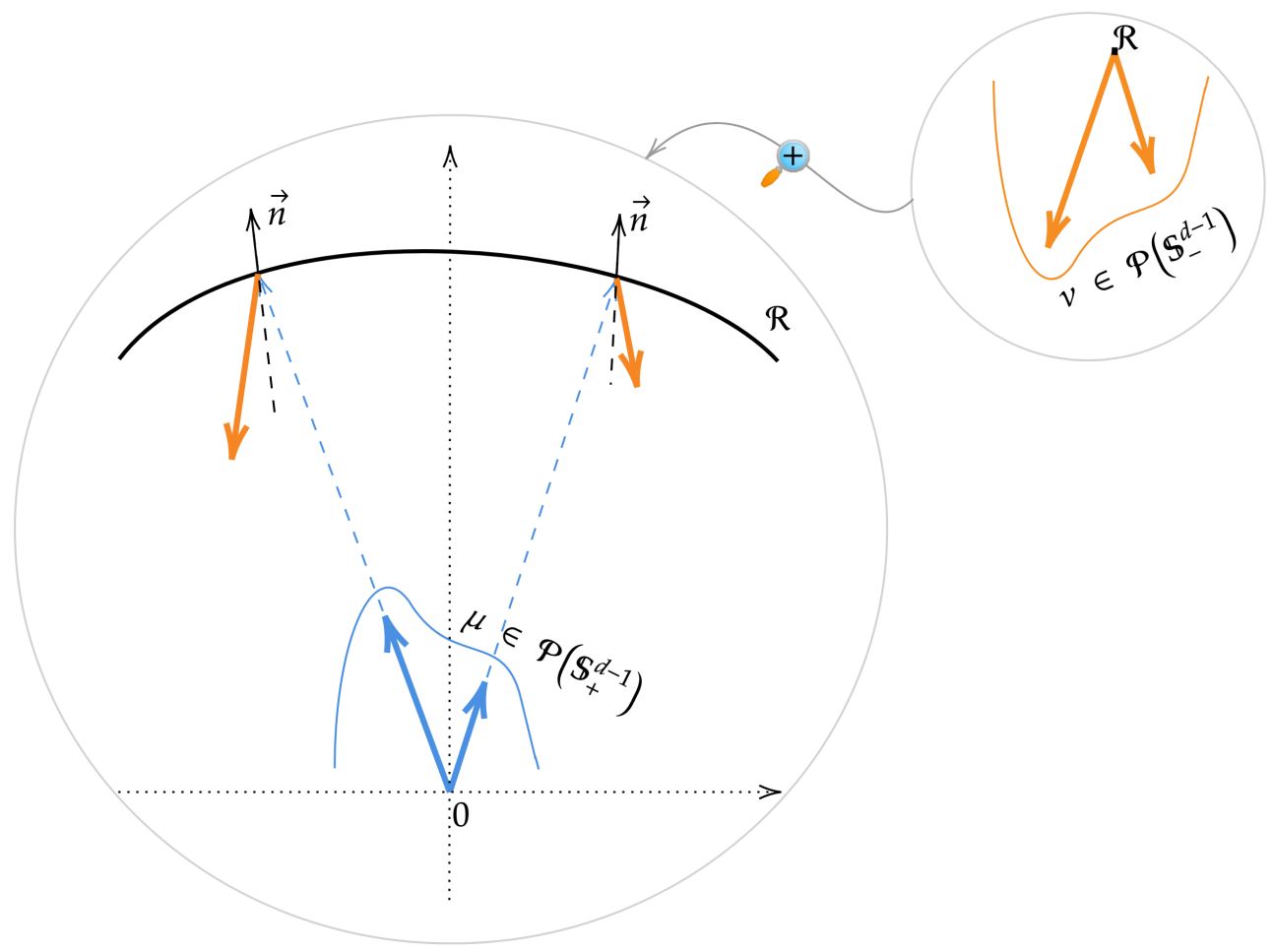

Figure 1: Point Source (PS) reflector.

Before moving to the resolution of the Finite source problem, let us summarize a few results on the easier Point Source Reflector problem formulated below:

Find $\mathcal{R}$ or $\rho$ (see $(1))$ given $\left(\mu_{0}, \nu\right)$ in the $R=\infty$ (or $\eta=0$ ) regime. (PS) 
Example 1. The simplest example of a point source solution is the parabola with focal at $0_{R^{2}}$ and focal direction $\hat{y}_{0}: \rho\left(x_{0}\right)=C /\left(1-\hat{x}_{0} \cdot \hat{y}_{0}\right)$. The constant $C$ fixes the focal distance and for any illuminance $\mu_{0}$ all the light illuminates the single direction $\hat{y}_{0}$.

A second example is the "identity" reflector $\rho\left(x_{0}\right)=C$ a constant, which maps any $\mu_{0}$ to its flipped mirror image $\nu(y)=\mu_{0}(y-\pi)$.

Mathematically, (PS) is equivalent to finding a ray mapping $T_{0}: x_{0} \in$ $X_{0} \mapsto y_{x_{0}} \in Y$ from the source point $O_{\mathbb{R}^{2}}$ and between incoming/outgoing angles, satisfying the following two conditions:

- Descartes-Snell's reflection law on the reflector :

$$
\hat{y}_{x_{0}}=\hat{x}_{0}-2\left(\hat{x}_{0} \cdot \hat{n}_{0}\left(x_{0}\right)\right) \hat{n}_{0}\left(x_{0}\right) \text { or } y_{x_{0}}=2 n_{0}\left(x_{0}\right)-\left(x_{0}+\pi\right),
$$

where $\hat{n}_{0}\left(x_{0}\right)$ is the outer normal to the reflector $\mathcal{R}$ at point $\hat{x}_{0} \rho\left(x_{0}\right)$ and $(.$.$\left.) the Euclidean scalar product in \mathbb{R}^{d}\right)$. Following our angle notation convention, $n_{0}\left(x_{0}\right)$ will be the angle of the normal at the reflector point $\mathbb{R}\left(x_{0}\right)$.

- Local Energy conservation by the map, ensuring that the reflection indeed creates the desired illumination from the illuminance:

$$
\begin{aligned}
& \left(\nu \circ T_{0}\right)\left|\frac{\partial T_{0}}{\partial x_{0}}\right|=\mu_{0} \text { if } T_{0} \text { is smooth or } \\
& \int \phi d \nu=\int \phi \circ T_{0} d \mu_{0}, \forall \phi \in \mathcal{C}\left(S_{+}^{1}\right) \text { in its weak form. }
\end{aligned}
$$

The operation in (4) is often denoted $\nu=T_{0} \# \mu_{0}$ ( $T$ "pushes forward" $\mu_{0}$ to $\left.\nu\right)$.

An elegant variational formulation linked to Optimal Transportation has been proposed and studied in [1]: Assuming that the support of respectively $\mu_{0}$ and $\nu_{0}$, are connected domains subset of respectively of $\mathbb{S}_{+}^{d-1}$ and $\mathbb{S}_{-}^{d-1}$ (as in section 1.1), then there exists an unique (up to dilations) reflector achieving the desired light transfer given by the radius function

$$
\rho=e^{f_{0}},
$$

where $f_{0}$ is the, so called Kantorovich potential, unique (up to a constant) solution of 


$$
\left(f_{0}, g_{0}\right):=\operatorname{Arg} \sup _{\{(f, g) \in \mathcal{D}\}}\left\langle f, \mu_{0}\right\rangle_{X_{0}}+\left\langle g, \nu_{0}\right\rangle_{Y}
$$

with

$$
\mathcal{D}=\left\{(f, g) \in \mathcal{C}\left(X_{0}, \mathbb{R}\right) \times \mathcal{C}(Y, \mathbb{R}) \mid f\left(x_{0}\right)+g(y) \leq c\left(x_{0}, y\right)\right\}
$$

and $\langle f, \mu\rangle_{X}$ being the notation for the duality product between continuous functions and probability measures over $X$.

This is the well know dual formulation of the Monge-Kantorovich problem (see [2], Chap. 2). When $c$ is the "reflector cost":

$$
\begin{aligned}
c\left(x_{0}, y\right) & \left.=-\log \left(1-\hat{x}_{0} \cdot \hat{y}\right)\right) \\
& =-\log \left(1-\cos \left(x_{0}-y\right)\right)(\text { for } d=2),
\end{aligned}
$$

the optimal transport map:

$$
\left.T_{0}: x_{0} \rightarrow y_{x_{0}}:=\left\{y \mapsto \nabla_{x} c\left(x_{0}, y\right)\right\}\right\}^{-1}\left(\nabla_{x} f_{0}\left(x_{0}\right)\right),
$$

arising from the optimality conditions of (6), satisfies (3) and (4).

The crucial point in [1] and [3], obtained independently, was to establish that, for the reflector cost (8), the optimal map $x_{0} \mapsto y_{x_{0}}=T_{0}\left(x_{0}\right)$ satisfies the specular reflection law (3) and therefore models the incoming/outgoing angles of a ray hitting the convex reflector constructed with the optimal potential $f_{0}$ :

$$
\mathcal{R}_{f_{0}}:=\left\{\hat{x}_{0} e^{f_{0}\left(x_{0}\right)}, x_{0} \in X_{0} \text { s. t. } e^{f\left(\frac{\pi}{2}\right)}=R\right\} .
$$

This result is not straightforward and comes from algebraic computations and the interpretation of $\mathcal{R}_{f_{0}}$ as an envelope of parabolae (see (37) in the annex). As the optimal map $T_{0}$ also satisfies the conservation of energy (4), we directly are in the geometrical optics approximation of light propagation. This Optimal Transportation problem is a well posed variational formulation for finding a ray mapping satisfying the illuminance/illumination constraints discussed in the introduction for a single point source. It will be used as a parameterization tool for the more complicated finite source inverse problem (next subsection).

Remark 1. As already mentionned the solutions to (6) are unique up to a constant: $\left(f_{0}+C, g_{0}-C\right)$ are optimal for any $C$. For the point source reflector problem, this simply means that the distribution in the angular far-field can be reached by any dilation of the optimal reflector. Hence the need to fix the constant $R$ in (10). 
We gather in the following proposition a few useful properties of these point source solutions (the proofs are postponed to the annex).

\section{Proposition 1 (On the Point source Optimal Transportation reflector).}

(i) $\mathcal{R}_{f_{0}}, f_{0}$ solution of (6) is a strictly convex curve (surface for $d=3$ ).

(ii) If $\mu_{0}$ and $\nu_{0}$ are Hoelder continuous and bounded below by a positive constant, $Y$ c-convex then the Ma-Trudinger-Wang regularity theory applies [4] [5], $f_{0}$ is bounded in $C^{2, \alpha},(0<\alpha<1)$.

(iii) The angle of the normal to $\mathcal{R}_{f_{0}}$ at point $x_{0}, n_{0}\left(x_{0}\right)$ satisfies: $\tan \left(n_{0}\left(x_{0}\right)\right)=$ $\partial_{x_{0}} f_{0}\left(x_{0}\right)$, in particular the strict monotony of $x_{0} \rightarrow n_{0}\left(x_{0}\right)$ induced by (i) carries over to $x_{0} \rightarrow \partial_{x_{0}} f_{0}\left(x_{0}\right)$.

\subsection{The Finite Source Problem}

The illuminance patch $S$ is now for a finite $R$ (see illuminance in section 1.1). The finite source problem is:

$$
\text { Find } \mathcal{R} \text { or } \rho \text { (see (1)) given }\left(\mu_{S}, \nu\right) \text {, }
$$

(see the illuminance/illumination section 1.1 for the notations).

In section 2, we will model this problem as a collection of point source problems:

For each $\mu_{s}, s \in S, T_{s}: x_{s} \in X_{s} \mapsto y_{x_{s}} \in Y$ is the ray reflection mapping on $\mathcal{R}$ from the source point $O_{s}=(0, s), s \in S$. The illumination from the extended source is given by the sum over the point source illuminations:

$$
\nu:=\int_{S} \nu_{s} d s \text { where } \nu_{s}=T_{s} \# \mu_{s}, \forall s \in S,
$$

(see (4) for the definition of the pushforward).

Even in the simplest case where $S$ is composed of two point sources, problem (ES) is likely to be overdetermined. One reflector surface should map two distinct sources to the far-field. We can expect at each point on the reflector to receive two incoming rays while there is only one "control", 


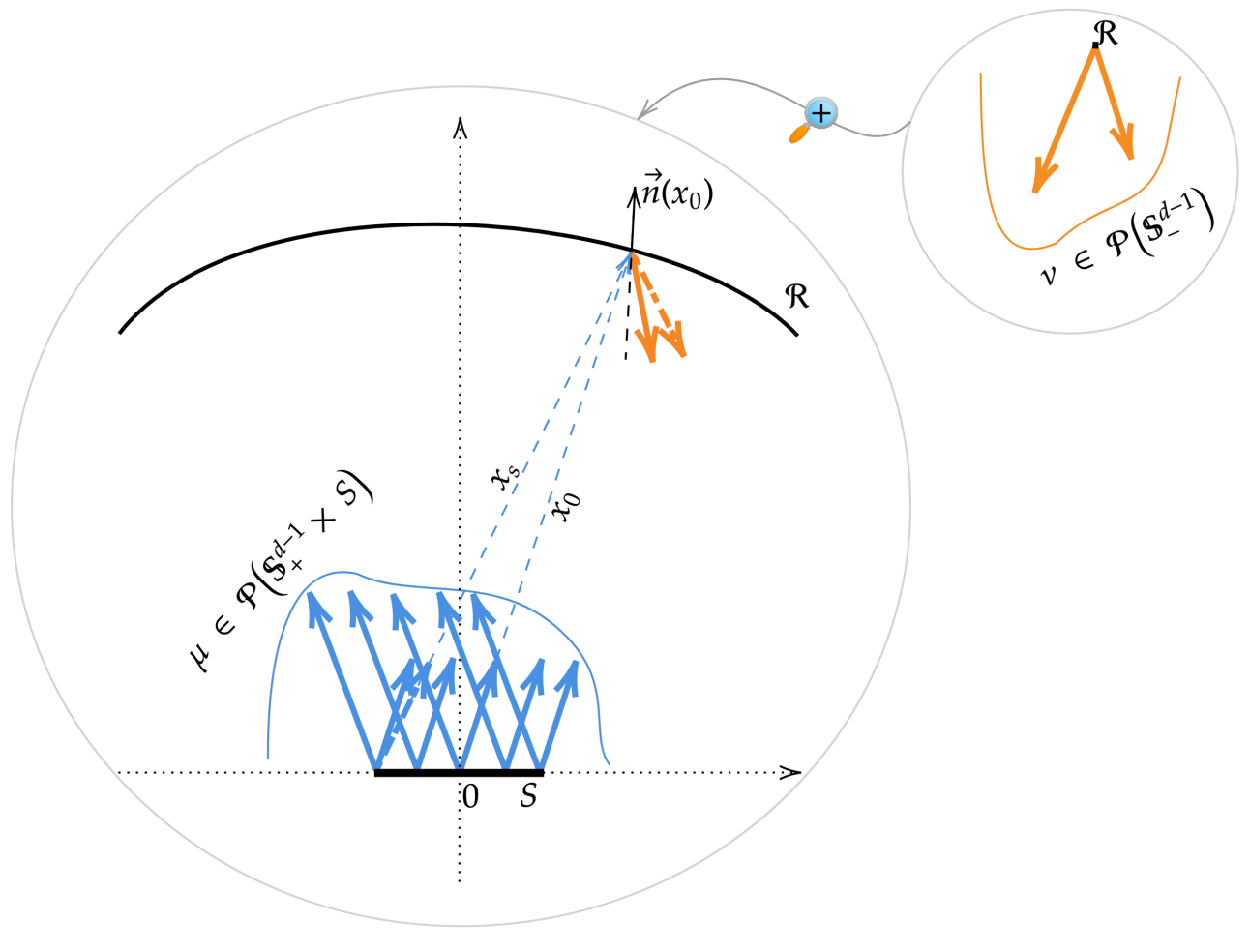

Figure 2: Finite source (ES) reflector. 
the normal of the reflector at that point. We also formally show (remark 2) that for a smooth and convex reflector the target illumination is related to a non-linear convolution of a point source target. As illustrated in the parabola example 1, see also figure 6, this implies smoothness and support constraints on the prescribed illumination distribution. We are not aware of any theoretical results providing information on the ill or well-posedness under some additional constraints on the data.

This naturally suggests a regularization approach as is customary for illposed non-linear inverse problems (see for instance [6] for a recent review). In this framework we need:

1. A parametric set $\mathcal{M}_{\Theta}$ of admissible reflectors: $\theta \in \Theta \rightarrow \mathcal{R}_{\theta} \in \mathcal{M}_{\Theta}$. This is the regularization part.

2. A forward map: $\mathcal{F}: \theta \in \Theta \rightarrow \nu_{\mathcal{R}} \in \mathcal{P}(Y)$, i.e. $\mu_{S}$ (the extended source) and $\theta$ given, compute the illumination produced by the reflector $\mathcal{R}_{\theta}$. This maps encodes the reflector optics and can be implemented using ray tracing.

3. A loss/merit function: $\mathcal{L}:\left(\nu, \nu^{\prime}\right) \in \mathcal{P}(Y) \times \mathcal{P}(Y) \mapsto \mathcal{L}\left(\nu, \nu^{\prime}\right) \in \mathbb{R}^{+}$ giving sufficient information on the "closedness" between $\nu$ and $\nu^{\prime}$. Ideally $\mathcal{L}$ should be a distance or at least satisfy $\mathcal{L}:(\nu, \nu)=0$ and $\nu^{\prime} \mapsto \mathcal{L}:\left(\nu^{\prime}, \nu\right)$ is positive and convex.

Then, assuming $\mathcal{F}$ and $\mathcal{L}$ are computable objects, the regularized solution to the finite source problem is the reflector corresponding to the following parameter (we drop the dependance in $\mu_{S}$, it remains fixed):

$$
\theta^{*}:=\operatorname{Arg} \inf _{\theta \in \Theta} J(\theta) \text {, where } J(\theta):=\mathcal{L}(\mathcal{F}(\theta), \nu) \text {. }
$$

The cost function $\theta \mapsto J(\theta)$ is bounded below by construction. If it is also continuous (or lower semi-continuous) and $\mathcal{M}_{\Theta}$ is compact for the chosen topology, then $J$ has a minimizer. The global minimizer $\theta^{*}$, if we can compute it, gives the "best" reflector $\mathcal{R}_{\theta^{*}}$ within $\mathcal{M}_{\Theta}$ and $J\left(\theta^{*}\right)$ is a measure of its quality.

\section{State of the art}

A parameterization of $\mathcal{R}$ in the ambient space, i.e. $\mathcal{M}_{\Theta}$ could be any discrete parameterization of curves (Splines, Bezier ....) where $\theta$ would be control points and tangents for example. Then the modifications happen 
by local deformations of the reflector(see e.g. [7]). In [8] spline parameterization is used to apply optimization methods for minimizing the $L_{2}$ norm between the desired target and the outcome of the forward map. Although such parameterizations are associated with extra complications when trying to guarantee some desired properties of the reflector, e.g convexity/concavity. The point source problem is sometimes used to approach the extended source problem. For example, this is the approach followed in [9] (see also [10], [11] and the references therein). The reflectors is then updated by pointwise modifications of the point source problem densities.

\section{Contribution}

We also use the point source problem (PS) to build a parameterization of the reflector. This is explained in section 2. We leverage the well-posedness and regularity of its optimal transport formulation to build a continuous loss (section 3). In the same section we also give a variational interpretation of the Gold deconvolution method used in this context by [9]. From the computational point of view, several optimal transport solvers are available. We follow the entropic regularization approach proposed in our previous work [12] which provides additional smoothness. Numerical results are given in section 4 . We discuss the numerical and theoretical open questions in the conclusion.

\section{Construction of the forward map}

\subsection{Point source parameterization of the Reflector}

The Parametric set is chosen as $\mathcal{M}_{\Theta}=\mathcal{P}\left(\mathbb{S}_{+}^{d-1}\right)$ and the parameters $\theta$, relabelled $\nu_{0}$ spans the set of illuminations for the point source problem (PS). We also need to specify an illuminance for this point-source optimal transport problem. It is chosen to remain fixed but it could also be part of the parameterization (this is not investigated in this paper). We have been using the sum of the $\mu_{s}$ (also denoted $\mu_{0}$ by abuse of notation):

$$
\mu_{0}(.)=\int_{S} \mu_{s}(.) d s .
$$

The reflector $\nu_{0} \rightarrow \mathcal{R}_{f_{0}}$ is uniquely constructed from $f_{0}$ the solution of (6) where $\nu=\nu_{0}$. The constant is fixed as in (1). This choice constrains the reflector to be a strictly convex curve. It desirable with respect to engineering constraints but it is also crucial to prove the following re-parameterization 
using the angle parameters $x_{s} \in X_{s}$ from the other point sources $O_{s}:=$ $(s, 0), s \in[-\eta, \eta]$ on $S:$

Proposition 2 (Re-parameterization of $\mathcal{R}_{f_{0}}$ ). Let us assume that $X_{0}=$ $X_{s}=\mathbb{S}_{+}^{1}$ for all $s$ and $\eta<\frac{R}{2}$. Then, for all $s \in S$ there exists a function $f_{s}: X_{s} \rightarrow \mathbb{R}^{+}$(see figure 3a) such that:

(i) The following re-parameterization of the reflector holds :

$$
\mathcal{R}_{f_{0}}=\mathcal{R}_{f_{s}}:=\left\{\hat{x}_{s} e^{f_{s}\left(x_{s}\right)}, x_{s} \in X_{s}\right\} .
$$

where $\hat{x}_{s}$ is the unit vector in the direction $x_{s}$ from $O_{s}$.

(ii) The inner normal angle in the (13) parameterization, denoted $n_{s}\left(x_{s}\right)$ is given by

$$
\tan \left(n_{s}\left(x_{s}\right)\right)=\frac{\partial_{x_{s}} f_{s}\left(x_{s}\right) \cos \left(x_{s}\right)+\sin \left(x_{s}\right)}{\cos \left(x_{s}\right)-\partial_{x_{s}} f_{s}\left(x_{s}\right) \sin \left(x_{s}\right)}
$$

(iii) The map $f_{0} \mapsto f_{s}$ is continuous for the $\mathcal{C}^{1}\left(\mathbb{S}_{-}^{1}\right)$ topology.

\section{Proof 1. See annex.}

Proposition 2 is used in the next section to establish the continuity of the forward map. Remark 2 also shows formally that assuming the existence of a smooth forward map, $\nu$ the prescribed illumination is a non-linear convolution of $\nu_{0}$.

\subsection{The forward map}

As sketched in section 1.3 and (11), the extended source forward/ray tracing map can be decomposed as follows:

$$
T_{s}: x_{s} \rightarrow y_{x_{s}}:=2 n_{s}\left(x_{s}\right)-\left(x_{s}+\pi\right)
$$

is the reflection ray map from $O_{s}$ onto $\mathcal{R}_{f_{s}}$ the re-parameterization given in proposition 2 .

The resulting illumination is given by:

$$
\nu_{\mathcal{F}}:=\mathcal{F}\left(\nu_{0}\right)=\int_{S} \nu_{s} d s \text { where } \nu_{s}=T_{s} \# \mu_{s}, \forall s \in S .
$$


We summarize the forward map $\nu_{0} \rightarrow \nu_{\mathcal{F}}=\mathcal{F}\left(\nu_{0}\right)$ :

$A: \quad \nu_{0} \mapsto f_{0}$ (solution of (6), $\mu_{0}$ fixed and $\left.\nu=\nu_{0}\right)$.

$B: f_{0} \mapsto\left\{f_{s}\right\}_{s \in S}$ (proposition $2(\mathrm{i})$ ) .

$C:\left\{f_{s}\right\}_{s \in S} \mapsto \nu_{\mathcal{F}}:=\mathcal{F}\left(\nu_{0}\right)$ (formulae (15) and (16)).

And we have:

Theorem 1. Under the assumptions of proposition 1-2, $\mathcal{F}$ is continuous for the weak topology in $\mathcal{H} \in \mathcal{P}\left(S_{+}^{1}\right)$, the set of probability distributions with Hoelder continuous and bounded below densities.

Proof 2. The restriction $\nu_{0} \in \mathcal{H}$ is needed to define the reparameterization and define $\mathcal{F}$ itself. See the annex for the rest of the proof.

Remark 2 ( $\mathcal{F}$ is formally a non-linear convolution). We start back from (16) and assume (to simplify the exposition) that $\mu_{s}=\frac{1}{2 \eta} \mu_{0}$ for all s. Meaning that the radiation pattern is identical for all points on the finite source. We ignore the normalization constant (again to simplify the notation) and we get, $\forall y \in Y$ :

$$
\begin{aligned}
\nu_{\mathcal{F}}(y) & =\int_{S} T_{s} \# \mu_{0}(y) d s \\
& =\int_{S} \mu_{0}\left(T_{s}^{-1}(y)\right)\left(\partial_{x_{s}} T_{s \mid T_{s}^{-1}(y)}\right)^{-1} d s \\
& =\int_{S} \nu_{0}\left(T_{0} \circ T_{s}^{-1}(y)\right) \partial_{x_{0}} T_{0 \mid T_{s}^{-1}(y)}\left(\partial_{x_{s}} T_{s \mid T_{s}^{-1}(y)}\right)^{-1} d s .
\end{aligned}
$$

We now assume that for a fixed $y$ the mapping $\left.s \mapsto \mathcal{Y}_{y}(s)=T_{0} \circ T_{s}^{-1}(y)\right)$ (see figure $3 b$ ) is bijective from $S$ onto its domain and make the change of variable

$$
\nu_{\mathcal{F}}(y)=\int_{\mathcal{Y}_{y}(S)} \nu_{0}\left(y^{\prime}\right) \partial_{x_{0}} T_{0 \mid T_{\mathcal{Y}_{y^{\prime}}^{-1}(y)}^{-1}(y)}\left(\partial_{x_{s}} T_{\mathcal{Y}_{y^{\prime}}^{-1}(y) \mid T_{\mathcal{Y}_{y^{\prime}}^{-1}(y)}^{-1}(y)}\right)^{-1} d y^{\prime} .
$$

The formula is complex but shows that $\nu_{0}$ is convolved with a Kernel involving the Jacobians of the maps $T_{s}$ and $T_{0}$ :

$$
\mathcal{K}\left(y, y^{\prime}\right):=\partial_{x_{0}} T_{0 \mid T_{\mathcal{Y}_{y^{\prime}}^{-1}(y)}^{-1}(y)}\left(\partial_{x_{s}} T_{\mathcal{Y}_{y}^{-1}\left(y^{\prime}\right) \mid T_{\mathcal{Y}_{y}^{-1}\left(y^{\prime}\right)}^{-1}(y)}\right)^{-1},
$$

depending on the reflector, hence also on $\nu_{0}$. The map $s \mapsto \mathcal{Y}_{y}(s)$ can be interpreted as follows (see also figure 3b) : given a reflection direction $y$ and 
a point $s$ on the finite source, find the shooting angle $x_{s}$ from that point. Then shoot a ray from the center source point $O_{0}$ with the same angle $x_{0}=x_{s}$ and record the outgoing angle. Given two outgoing angles $y$ and $y^{\prime}$, its inverse returns the coordinate on the finite source for which the shooting angle with $y^{\prime}$ reflection is the same as the shooting angle from $O_{0}$ yielding $y$. For instance $y=y^{\prime}$ gives $\mathcal{Y}_{y}^{-1}(y)=0$ and $\mathcal{K}(y, y)=1$.

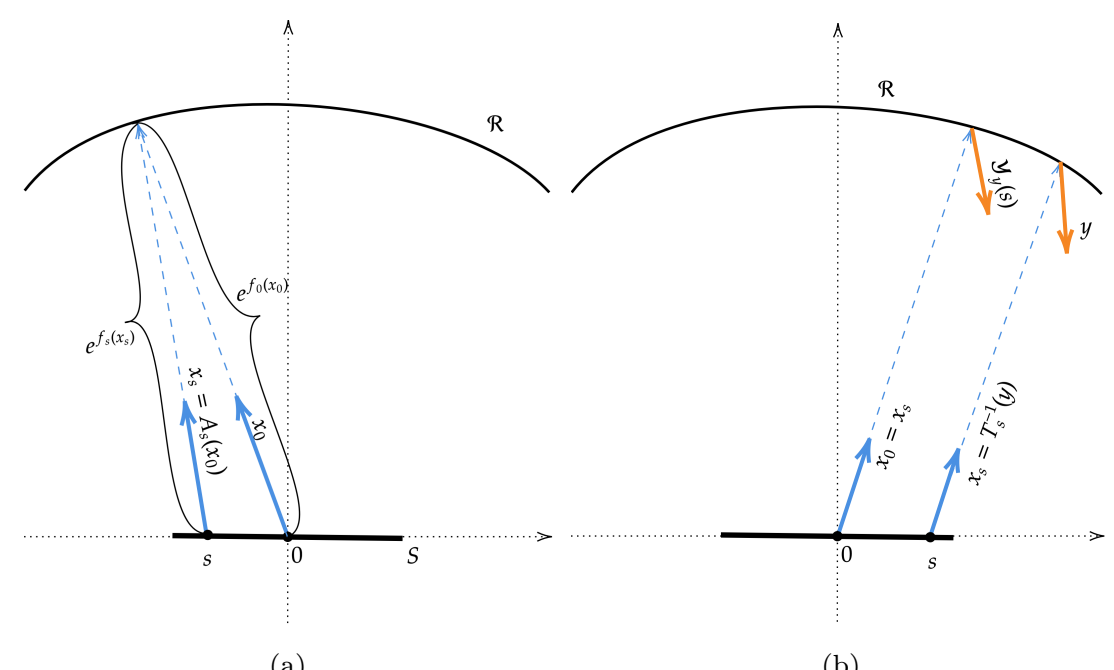

(a)

(b)

Figure 3:

(A): Reparametrization from $x_{0} \in X_{0}$ to $x_{s} \in X_{s}$. (B): The map $s \rightarrow \mathcal{Y}_{y}(s)$ defined as $T_{0} \circ T_{s}^{-1}(y)$.

Remark 3. In [10] a loose form of this convolution is used to solve some simple cases of the extended source problem, by iteratively approximating the convolution kernel $\mathcal{K}$

\subsection{Numerical resolution of (FM)-A}

The first task is to compute the point source reflector $\mathcal{R}_{f_{0}}$ or equivalently $f_{0}$. Several approaches are possible. Classical linear programming methods are too costly (see [13] Chap. 2). The Monge-Ampère/spline-collocation method proposed in [14] is rather technical and will not be discussed here. The semi-discrete approach is computationally efficient and could certainly be used here [15]. Other methods based on finite difference discretizations of the Monge-Ampère equation [16] [17] are also relevant. 
Finally, it is also possible to use the entropic regularization of (6) (actually, a variant called Sinkhorn divergence). This is the path chosen in this paper. It is summarized below. For a comprehensive presentation of entropic Optimal Transportation see [13], Chap. 4. The application to the point source reflector problem is detailed in [12].

The entropic Optimal Transportation problem depends on a regularization parameter denoted $\varepsilon$ and consists in solving

$$
\begin{gathered}
\left(f_{0, \varepsilon}, g_{0, \varepsilon}\right):=\operatorname{Arg} \sup _{(f, g)} \mathcal{D}_{\left(\mu_{0}, \nu_{0}\right)}^{\varepsilon}(f, g) \\
\mathcal{D}_{\left(\mu_{0}, \nu_{0}\right)}^{\varepsilon}(f, g):=\left\langle f, \mu_{0}\right\rangle_{X_{0}}+\left\langle g, \nu_{0}\right\rangle_{Y}-\varepsilon\left\langle e^{\frac{1}{\varepsilon}(f \oplus g-c)}, \mu_{0} \otimes \nu_{0}\right\rangle_{X_{0} \times Y} .
\end{gathered}
$$

The additional regularization on the right acts as a barrier function for the Kantorovich constraint $f \oplus g-c \leq 0$ (see (6)). Problem (19) is unconstrained, strictly concave and $\left(f_{0, \varepsilon}, g_{0, \varepsilon}\right)$ are the (unique) solutions of the optimality conditions system:

$$
f_{0, \varepsilon}=L S E_{\nu_{0}, Y}^{\varepsilon}\left(g_{0, \varepsilon}\right) \quad g_{0, \varepsilon}=L S E_{\mu_{0}, X_{0}}^{\varepsilon}\left(f_{0, \varepsilon}\right),
$$

where we introduced the $L o g / S u m / \operatorname{Exp}(L S E)$ operator taking a function on $Y$ returning a function on $X_{0}$ (remember $c$ is a function defined on $X_{0} \times Y$ ):

$$
L S E_{\rho, Y}^{\varepsilon}: g \rightarrow-\varepsilon \log \left(\left\langle e^{\frac{1}{\varepsilon}(g-c)}, \rho\right\rangle_{Y}\right) .
$$

The $C^{\infty}$ regularity of $c$ carries over through formula (20) to the potentials.

The popular iterative (in $k$ ) Sinkhorn algorithm is the following relaxation of (20) (corresponding to a coordinate ascent maximization):

$$
f_{0, \varepsilon}^{(k)}=L S E_{\nu_{0}, Y}^{\varepsilon}\left(g_{0, \varepsilon}^{(k-1)}\right) \quad g_{0, \varepsilon}^{(k)}=L S E_{\mu_{0}, X_{0}}^{\varepsilon}\left(f_{0, \varepsilon}^{(k)}\right) .
$$

In practice, the illuminance/illumination are discrete probability measures:

$$
\mu_{0}=\sum_{i=1}^{N_{X_{0}}} \mu_{0, i} \delta_{x_{0, i}} \quad \nu_{0}=\sum_{j=1}^{N_{Y}} \nu_{0, j} \delta_{y_{j}}
$$

and $X_{0}=\left\{x_{0, i}, i=1 . . N_{x_{0}}\right\}, Y=\left\{y_{j}, j=1 . . N_{Y}\right\}$. The vector coefficients $\left(\mu_{0, i}\right) \mathrm{s}$ and $\left(\nu_{0, j}\right) \mathrm{s}$ are positive and sum to 1 . For a fixed discretization $N_{0, Y}$, the convergence both in $k$ and $\varepsilon$ hold [18]. The canonical interpolation 
method for the potentials is to define the continuous $\left(\forall x_{0} \in \mathbb{S}_{+}^{1}\right)$ extension of $f_{0, \varepsilon}^{(k)}$ :

$$
\tilde{f}_{0, \epsilon}^{(k)}\left(x_{0}\right)=-\varepsilon \log \left(\left\langle e^{\frac{1}{\varepsilon}\left(g-c\left(x_{0}, \cdot\right)\right)}, \nu_{0}\right\rangle_{Y}\right)=-\varepsilon \log \left(\sum_{j} e^{\frac{1}{\varepsilon}\left(g-c\left(x_{0}, y_{j}\right)\right)} \nu_{0, j}\right)
$$

Instead of (FM)-A, we will be using:

$$
A_{\varepsilon}: \quad \nu_{0} \mapsto \tilde{f}_{0, \varepsilon}^{(K)}
$$

were $K$ is a fixed final iteration for $(22)$. This defines a smooth $\left(C^{\infty}\right)$ reflector $\mathcal{R}_{\tilde{f}_{0, \varepsilon}^{(K)}}$.

The strict convexity of the entropic reflector thus constructed is not guaranteed anymore and therefore also proposition 2. The use of Sinkhorn divergence is a simple method to correct the bias induced by the entropic regularization method [19]. It amounts to :

$$
f_{S D}=f_{0, \varepsilon}-f_{\mu_{0}, \varepsilon}
$$

where $f_{\mu_{0}, \varepsilon}$ solves the symmetric entropic Optimal Transportation problem

$$
f_{\mu_{0}, \varepsilon}:=\operatorname{Arg}_{\sup _{f}} \mathcal{D}_{\left(\mu_{0}, \mu_{0}\right)}^{\varepsilon}(f, f),
$$

$\left(\mathcal{D}^{\varepsilon}\right.$ is defined in (19)) and (25) can be formally understood as a first-order smooth correction for small $\varepsilon$. It can be solved using a symmetric version of Sinkhorn (22). There is no rigorous result assessing the accuracy of this correction but experiments suggest that the correction is close to the non entropic $\varepsilon=0$ solution while retaining the smoothness of the entropic potentials. See [12] for more details on this method. In practice, we use $\mathcal{R}_{f_{S D}}$ and assume proposition 2 is satisfied.

\subsection{Illumination through Ray Tracing}

(FM)-B and (FM)-C are performed simultaneously through the computation of reflections onto $\mathcal{R}$. This process is known as Ray Tracing in the optics community. Here we discuss two numerical approximations. 


\subsubsection{Forward Ray Tracing}

The first, to which we will refer as "forward ray tracing", is commonly used in the optics community. We assume that the $\left\{\mu_{s}\right\}_{s \in S}$ are given as discrete measures $\mu_{s}:=\sum_{i=1}^{N_{s}} \mu_{s, i} \delta_{x_{s, i}}$, each family $\left\{x_{s, i}\right\}_{i=1 . . N_{s}}$ being a discretization of $\mathbb{S}_{+}^{1}$. We also assume $S$ is a discrete set and the positive coefficients $\mu_{s, i}$ are satisfying the energy conservation

$$
\sum_{s \in S} \sum_{i=1}^{N_{s}} \mu_{s, i}=1 .
$$

Based on (15) and (16) the finite source forward ray tracing produces the illumination:

$$
\nu_{\mathcal{F}}=\sum_{s \in S} \sum_{i=1} \mu_{s, i} \delta_{y_{s, i}}, \text { where } y_{s, i}=T_{s}\left(x_{s, i}\right)
$$

The computation of $x \mapsto T_{s}(x)$ requires the normal $n_{s}$ to $\mathcal{R}$ (see proposition 2 (ii)) at the intersection between the ray shot from $O_{s}$ in the direction $\hat{x}$ and the reflector curve. In the continuous case, this intersection is always well defined according to proposition 2. This approach is not limited by the number of rays that can be shot, except for the computational time. Assuming enough resources, it allows to accurately approximate the continuous illumination. In practice, the intersection between each ray and some interpolation of the reflector needs to be computed, together with the normal

of the reflector at the intersection point, computation for a large number of rays becomes time-consuming even for the simplest, linear (bi-linear for $\mathrm{d}=3$ ) interpolations.

\subsubsection{Backward Ray Tracing}

In order to speed up the computations, we also use another method, which we will refer to as "backward ray tracing". In this approach, we do not fix the sampling of the source, but instead we assume that we have access to a continuous analytic density for $\mu_{s}$ and hence we construct a sampling corresponding to a prescribed discrete support in angle. The angle discretization $\left\{x_{0, i}\right\}_{i}$ of $X_{0}$ for the $0_{\mathbb{R}^{2}}$ center source point is also the support of the discrete reflector. Normals at those points can be computed by taking the gradient of the canonical continuous extension (23). This can be implemented using automatic differentiation tools. 
Then, for all $(s, 0) \in S$ and using the transform $\mathcal{A}_{s}$ (see the proof of Proposition 2 for the definition), we can get a (not necessarily uniform ) discretization $\left\{x_{s, i}\right\}_{i}:=\left\{\mathcal{A}_{s}\left(x_{0, i}\right)\right\}_{i}$ of $X_{s}$. In order to account for the nonuniformity of this discretization, we perform a piecewise constant approximation of the the density on this grid. For $\mathrm{d}=2$, define $\Delta_{s, i}:=\left(x_{s, i+1}-x_{s, i-1}\right) / 2$, and use as an empirical approximation:

$$
\mu_{S} \simeq \sum_{s} \sum_{i} \Delta_{s, i} \mu_{s}\left(x_{s, i}\right) \delta_{x_{s, i}} .
$$

Backward ray tracing produces the illumination:

$$
\nu_{\mathcal{F}}=\sum_{s \in S} \sum_{i=1} \Delta_{s, i} \mu_{s}\left(x_{s, i}\right) \delta_{y_{s, i}}, \text { where } y_{s, i}=T_{s}\left(x_{s, i}\right) .
$$

There are, of course, various ways to improve this simple strategy by optimizing the weights using different estimators. The discrete illumination follows from the same equation (27) but the number of rays is fixed and the same as the discretization of the problem and there is no intersection with the reflector point to compute. The drawbacks of this approach lays in the quality of the sampling which cannot be controlled directly. In practice (and for $d=2$ ) it does not seem to be a problem.

\subsubsection{The "Binning" technique}

The ray tracing methods generate discrete point cloud $\left\{y_{s, i}=T_{s}\left(x_{s, i}\right)\right\}_{s, i}$ distributions in the angle space $\mathbb{S}_{-}^{d-1}$ with weights $w_{s, i}$. It could be desirable to have this distribution on a grid or another set of points denoted here $\left\{z_{k}\right\}$ (e.g. to have the reflected distribution in the form of a pixelized picture, or because the desired target density is given on such grid: $\nu=\sum_{k} \nu_{k} \delta_{z_{k}}$ and one wishes to do a point-wise comparison).

To achieve this, we define "bins" $\left\{B_{k}\right\}$, that is a disjoint cells covering of $Y$ with centers $z_{k}$. For $d=2$ and assuming the $z_{k}$ are ordered, we use $B_{k}=$ $\left[\frac{z_{k}+z_{k-1}}{2}, \frac{z_{k}+z_{k+1}}{2}\right)$. For $d=3$ the shape of the bins can be more complicated, but if $z_{k}$ are induced by some structured grid, this structure will dictate what the shape should be.

The "binned" approximation is constructed by summing the weights of all rays falling into an each bin $B_{k}$ to obtain $\bar{\nu}_{\mathcal{F}}\left(y_{j}\right):=\sum_{\left\{i: T\left(x_{i}\right) \in B_{j}\right\}} w_{i}$. The final discrete distribution $\bar{\nu}$ is usually noisy and is smoothed by a Gaussian convolution kernel, with standard deviation $\sigma=5 / N$ (where $\mathrm{N}$ is a number 
of "bins" used), which averages the values of neighbor bins, and results into a smoother distribution. The choice $\sigma=5 / N$, governing the width of the smoothing window, was made empirically.

\section{The Loss function}

\subsection{The Optimal Transport Loss}

The theory and practice of machine learning heavily relies on measuring discrepancies between discrete measures (weighted point clouds or histograms). A first option is to use again the Sinkhorn Divergence Loss but this time for its good statistical and regularity properties [13] [20] when applied to empirical estimators of probability density function. It is defined as:

$$
\begin{aligned}
\mathcal{L}_{S D}\left(\nu_{\mathcal{F}}, \nu\right):= & \mathcal{D}_{\left(\nu_{F}, \nu\right)}^{\varepsilon}\left(f_{0, \varepsilon}, g_{0, \varepsilon}\right)- \\
& \frac{1}{2}\left(\mathcal{D}_{\left(\nu_{\mathcal{F}}, \nu_{\mathcal{F}}\right)}^{\varepsilon}\left(f_{\nu_{\mathcal{F}, \varepsilon}} f_{\nu_{\mathcal{F}}, \varepsilon}\right)+\mathcal{D}_{(\nu, \nu)}^{\varepsilon}\left(f_{\nu, \varepsilon}, f_{\nu, \varepsilon}\right)\right),
\end{aligned}
$$

where $\left(f_{0, \varepsilon}, g_{0, \varepsilon}\right), f_{\nu_{\mathcal{F}}, \varepsilon}$ and $f_{\nu, \varepsilon}$ are the optimal potentials solutions of (19) and its symmetric variants (26).

In (8) the ground cost $c$ is chosen to build the optical reflection map from probability distributions (illumination and illuminance) supported on respectively the northern and southern hemisphere. In this section, our goal is to compare probability distributions (two illuminances associated with two reflectors) with the same support. The cost $c$ needs to be a distance on the support metric space, here $\mathbb{S}_{-}^{d-1}$. We used the squared Euclidean distance.

This loss has been studied in [19] where it is shown that $\nu_{\mathcal{F}} \mapsto \mathcal{L}_{S D}\left(\nu_{\mathcal{F}}, \nu\right)$ is convex, positive, differentiable and vanishes for $\nu_{\mathcal{F}}=\nu$. It is also continuous for the weak topology on measure.

We finally have :

Theorem 2. The global cost we minimize is $\nu_{0} \mapsto \mathcal{L}_{S D}\left(\mathcal{F}\left(\nu_{0}\right), \nu\right)$ : the composition of the the forward map (recall $\nu_{\mathcal{F}}=\mathcal{F}\left(\nu_{0}\right)$ ) and the loss (29). The loss parti s continuous for the weak topology on measures (like (29)) and the forward map is continuous on $\mathcal{H}$ (see Theorem 1), the compactness of $\mathcal{H}$ follows from Ascoli-Arzela and guarantees the existence of a minimizer. 
In order to get a dimensionless loss, we use the normalization :

$$
J\left(\nu_{0}\right):=\mathcal{L}\left(\mathcal{F}\left(\nu_{0}\right), \nu\right):=\mathcal{L}_{S D}\left(\mathcal{F}\left(\nu_{0}\right), \nu\right) / \mathcal{L}_{S D}(\mathcal{F}(\nu), \nu) .
$$

We recall that the desired prescribed target $\nu$ is the point source solution and the denominator cannot vanish as $\mathcal{F}(\nu)=\mathcal{K} \star \nu$ (see remark 2).

Figure 4 is a plot of the Loss value using a parametric family of point source target mixing two Gaussians with varying expectations:

$$
\left(t, t^{\prime}\right) \in[0,1]^{2} \mapsto \mathcal{L}\left(\mathcal{F}\left(\nu_{0}^{\left(t, t^{\prime}\right)}\right), \mathcal{F}\left(\nu_{0}^{\left(\frac{1}{3}, \frac{1}{3}\right)}\right),\right.
$$

where

$$
\nu_{0}^{\left(t, t^{\prime}\right)}=\mathcal{N}_{u(t), \frac{\pi}{21}}+\mathcal{N}_{v\left(t^{\prime}\right), \frac{\pi}{24}}, \quad u(t)=\frac{20 \pi}{16}+t \frac{2 \pi}{16}, \quad v\left(t^{\prime}\right)=\frac{26 \pi}{16}+t^{\prime} \frac{2 \pi}{16} .
$$

The graph is smooth and convex.
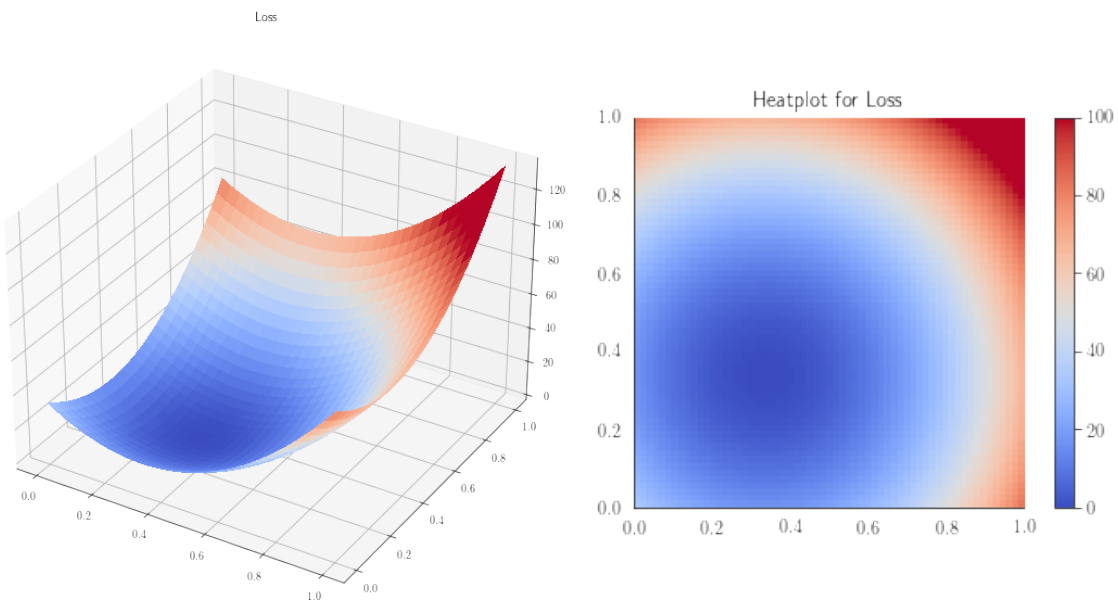

Figure 4: Graph plot of (31).

The optimal transport computations were implemented using the Geomloss package [21]. The desired illumination data $\nu$ is either discrete or sampled into a discrete empirical measure and the potentials are approximated through a finite number of Sinkhorn iterations (22). The differentiability with respect to $\nu_{\mathcal{F}}$, the desired property, is discussed in detail in [22]. The simplicity of the computational approach allows using the auto-differentiation 
algorithms embedded in modern software (we use Pytorch, for the full code see $[23])$.

Remark 4 (On the differentiability of $J$ ). While the Sinkhorn-Divergence potential $f_{S D}$ is differentiable with respect to $\nu_{0}$, the definition of the forward map itself depends on proposition 2 and the convexity of the reflector. This is only established for the non-entropic $\varepsilon=0$ reflector. In practice $f_{S D}$ (see (25)) is a good approximation of $f_{0}$ and we used the autodifferentiation and gradient based optimization methods of Pytorch without any difficulties.

\subsection{Gold deconvolution}

The analogy between the forward map and a non linear convolution $\nu=$ $\mathcal{K}_{\nu_{0}} \star \nu_{0}$ is explained in remark 2 . Interestingly, one of the heuristics proposed in the literature [9] to optimise $\nu_{0}$ is Gold de-convolution algorithm [24]: Iterate on $k$

$$
\nu_{0}^{(k+1)}:=\nu_{0}^{(k)}\left(\frac{\nu}{\mathcal{K}_{\nu_{0}^{(k)}} \star \nu_{0}^{(k)}}\right)^{\alpha}, \alpha>0 .
$$

This is easy to implement and, if convergent, we get $\nu=\mathcal{K}_{\nu_{0}^{(\infty)}} \star \nu_{0}^{(\infty)}$, the desired illumination. In the case of the reflector problem, $\mathcal{K}_{\nu_{0}}$ is not known explicitly but we can replace $\mathcal{K}_{\nu_{0}^{(k)}} \star \nu_{0}^{(k)}$ by the forward map $\mathcal{F}\left(\nu_{0}^{(k)}\right)$. Finally remark that $\nu_{0}^{(k+1)}$ has to remain a probability measure, this can simply be achieved by a re-normalization .

In the numerical result section we will compare its performance with the minimization of $\mathcal{L}_{S D}\left(\mathcal{F}\left(\nu_{0}\right), \nu\right)$ proposed in theorem 2. In this section, we point out (and it did not seemed to be mentioned in the literature) that (32) is also linked to the minimization of a loss. Let us consider the following sequence of minimization problems:

$$
\nu_{0}^{(k+1)}:=\operatorname{Arg} \inf _{\nu_{0}} \mathcal{K} \mathcal{L}\left(\nu_{0} \mid \nu^{(k)}\right)+\alpha \mathcal{K} \mathcal{L}\left(\mathcal{F}\left(\nu_{0}\right) \mid \nu\right),
$$

where $\alpha$ is a small positive "time" step and

$$
\begin{aligned}
\mathcal{K} \mathcal{L}\left(\nu_{\mathcal{F}} \mid \nu\right) & :=\left\langle\log \left(\frac{\nu_{\mathcal{F}}}{\nu}\right)-1, \nu_{F}\right\rangle+\langle 1, \nu\rangle \text { if } \nu_{F} \ll \nu \\
& +\infty \text { else }
\end{aligned}
$$


is the Kullback-Leibler divergence. It is also known as the relative entropy between $\nu$ and $\nu_{F}$, it is strictly convex, takes its minimum at $\nu$, and has an infinite slope at 0 . It forces $\nu_{F}$ to have the same support as $\nu$ and therefore relies on binning the rays (see section 2.4.3). Its Fréchet derivative in $\nu_{F}$ is formally given by $\left\langle\delta \mathcal{K} \mathcal{L}\left(\nu_{F} \mid \nu\right), \delta \nu\right\rangle=\left\langle\log \left(\frac{\nu_{F}}{\nu}\right), \delta \nu\right\rangle$.

For a small $\alpha,(33)$ maybe interpreted as a convex penalization of the direct minimization of the Kullback-Leibler loss:

$$
\mathcal{L}_{\mathcal{K L}}\left(\nu_{\mathcal{F}}, \nu\right):=\mathcal{K} \mathcal{L}\left(\mathcal{F}\left(\nu_{0}\right) \mid \nu\right) .
$$

If the resulting sequence $\left(\nu_{0}^{(k)}\right)$ converges it reaches a minimiser. The variational formulation (33) has strong analogies with the theory of Wasserstein Gradient Flows [2], Chap. 8 and some of the techniques developed in this context are likely to be applicable (for instance $\sum_{k} \mathcal{K} \mathcal{L}\left(\nu_{0}^{(k+1)} \mid \nu_{0}^{(k)}\right)$ is a convergent series).

Getting back to Gold method, the optimality condition for (33) leads to:

$$
\log \left(\frac{\left.\nu_{0}^{(k+1}\right)}{\nu_{0}^{(k)}}\right)=-\alpha \frac{\partial \mathcal{F}}{\partial \nu_{0}}\left(\nu_{0}^{(k+1}\right) \cdot \log \left(\frac{\mathcal{F}\left(\nu_{0}^{(k+1)}\right)}{\nu}\right) .
$$

This is a non-linear implicit system in $\nu_{0}^{(k+1)}, \frac{\partial \mathcal{F}}{\partial \nu_{0}}($.$) is a Jacobian operator$ or matrix. If we replace it by the identity matrix, (32) follows directly by taking the exponential of this expression and can be seen as a cheap explicit proxy of (33).

\section{Numerical Results}

\subsection{Experimental setting}

Reflector Height. The parameter $R$ first introduced in (1) "measures" how close the extended source problem approaches the point source problem. In our study It will vary between 1 and 9 .

Source Distribution and discretization. The source patch interval $S=[-0.5,0.5]$ will be fixed and the measure $\mu_{s}$ will always be uniform in 
$s$, that is, for all $s \in S, \mu_{s}=\mu_{0}$. Our approach is not limited to such measures but this assumption is the simplest and common for applications. For the source distribution $\mu_{0}$, plotted in figure 7 , we chose a distribution close to uniform within some angle opening and decays rapidly outside. To achieve these requirements, we take the sum of 16 Normal distributions, with means distributed uniformly within the interval $[9 \pi / 32,23 \pi / 32]$ and deviation $\sigma=\pi / 32$.

The number of points discretizing the angle spaces $X_{0, s}$ and the source interval $S$ denoted respectively $N_{A}$ and $N_{S}$ are chosen such that $\frac{\pi \cdot R}{N_{A}} \simeq \frac{2 \eta}{N_{S}}$ (the grid steps on the reflector or the source patch are of the same order). The number of rays $N$ shot is given foir backward ray tracing as $N=N_{A} \times N_{S}$. Setting $N$ and $R$ therefore also fixes the discretization size.

On our computer ${ }^{1}$ taking $N=5 \cdot 10^{6}$ and $R=5$, the $6 \mathrm{~GB}$ GPU memory was working at full capacity $(5.8$ out of $6 \mathrm{~GB})$ and the computation of the loss function with backward raytracing needs approximately 30 seconds. In comparison, it takes approximately 6 seconds for each iteration with $N=10^{5}$ and the used memory is approximately $1 \mathrm{~GB}$. This is the setting for all presented computations below.

Parameterization of $\nu_{0}$. The optimization variable is $\nu_{0} \in \mathcal{P}\left(\mathbb{S}_{+}^{1}\right)$. In practice it is parameterized using a classic machine learning method that guarantees that the optimization variable is a probability measure. The actual optimization variable is a vector $\lambda \in \mathbb{R}^{N_{A}}$ defined as

$$
\lambda_{i}:=\log \left(\nu_{0, i}\right)+\log \left(\sum_{i} e^{\nu_{0, i}}\right)
$$

where the $\left\{\nu_{0, i}\right\}$ s discretize $\nu_{0}$ and sum to 1 . The point source target entering the loss function is recovered by the inverse transform

$$
\nu_{0, i}:=\frac{e^{\lambda_{i}}}{\sum_{j} e^{\lambda_{j}}}
$$

Optimal Transportation computations. The implementation of the reflector computation and Sinkhorn divergence is based on Pytorch and the

\footnotetext{
${ }^{1}$ We ran the code on the laptop with a 64bit processor: Intel Core i7-8850H CPU @ 2.60GHz x 12 and GPU: Nvidia Quadro P3200 6GB with 1792 CUDA cores.
} 
Optimal Transportation platform Geomloss (available at [23]). The performance of the Sinkhorn algorithm and the bias induced by the entropic Optimal Transportation regularization is kown to depend on a blurring parameter $\varepsilon$. This is discussed in detail in [12] which suggests using $\varepsilon$ of order $1 / N_{A}$.

Optimization methods. We will compare three approaches: Explicit Gradient Descent adjusting the gradient step/learning rate experimentally (the gradient is obtained using Pytorch autodifferentiation), Adam algorithm [25] as implemented in Pytorch and we also implemented Gold method (32). We use a learning rate $l r=50$ for gradient descent, and $l r=0.1$ for Adam algorithm. Also, for Gold's method, we use the power parameter $\alpha=0.5$, which plays a similar role as the learning rate. Unless otherwise stated, we will always intitialize with $\nu_{0}=\nu$ the prescribed target distribution (this is also the solution for $R=+\infty)$.

\subsection{Dirac Targets and the convolution effect}

We start with a test case that illustrates the convolution effect (remark 2) and helps interpret more general solutions. As recalled in example 1, the simplest point source reflector is the parabola mapping any point (the focal point) source distribution to the direction of the focal axis. We use a Dirac target distribution $\nu=\delta_{3 \pi / 2}, R=5$. We use backward ray tracing and Adam optimization. Figure 5 compares the optimization with two initialization : $\nu_{0}=\nu$ the dirac mass itself and the normal distribution $\mathcal{N}_{\frac{3 \pi}{2}}, \frac{\pi}{41}$. We do not represent the reflector as it visuallly does not give much information. Instead, we plot (left) the "optimal" point source target parameterization of the reflector $\nu_{0}$ generated by the optimization and in dashed lines the initialization itself. The dirac initialization is stationary and the Gaussian converges to the Dirac solution (right). The convolution effect of the finite source onto the parabolic reflector is observed (center). Rays are binned as explained in section 2.4.3.

We can also illustrate the convolution effect by playing with the parameter $R$. Figure 6 we show the target distribution generated from the reflection of the finite source for parabolic reflectors with focal direction angles $\frac{5 \pi}{4}, \frac{3 \pi}{2}$ and $\frac{7 \pi}{4}$, and increasing heights $R 1,3,5,7$ and 9 . When larger, we approach the point source regime with a Dirac target distribution. 

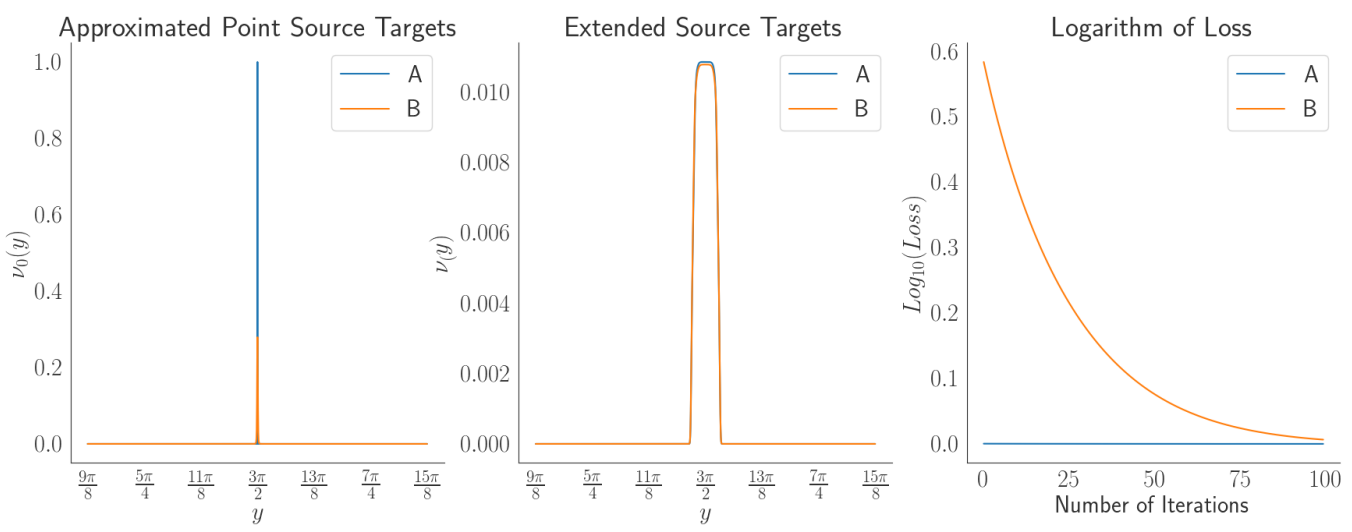

Figure 5: Dirac target distribution, two different initializations. (A): Initialized by the Dirac distribution. (B) Initialized by the Gaussian distribution. Left: final '"optimal " point source target parameterization of the reflector. Right: normalized loss function value along the optimization. Center: Target distribution simulated by ray tracing on the reflector generated by the optimization.

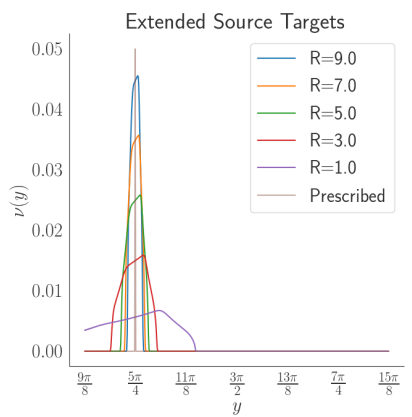

(a) $y=\frac{5 \pi}{4}$

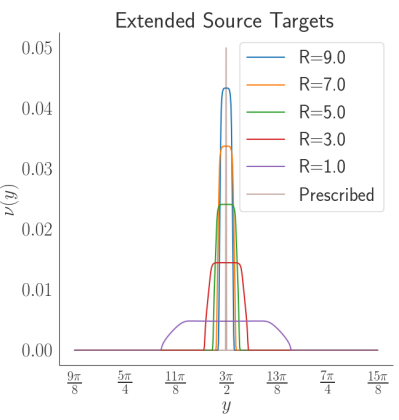

(b) $y=\frac{3 \pi}{2}$

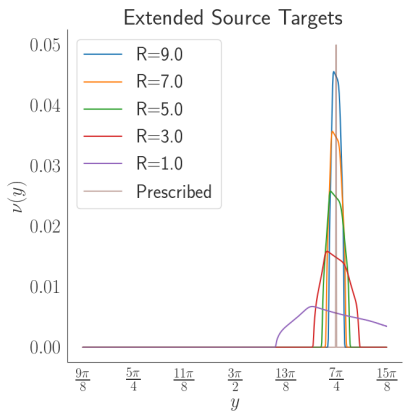

(c) $y=\frac{7 \pi}{4}$

Figure 6: Finite source reflection on parabolae with different focal axis and heights $R$. 


\subsection{Comparison of optimization methods}

Here we will present a comparison of the optimization methods (Adam, Gradient descent, Gold) for the following test cases (see figure 7) with a reflector height $R=5$ and backward ray tracing.

Test Case 1: "Uniform": $\nu=\frac{2}{\pi} \chi_{] \frac{5 \pi}{4}, \frac{7 \pi}{4}[}$ the characteristic function of the intervall $] \frac{5 \pi}{4}, \frac{7 \pi}{4}[$.

Test Case 2: Mixture of "Two Gaussians": $\quad \nu=\mathcal{N}_{\frac{3 \pi}{2}+\frac{\pi}{13}, \frac{\pi}{21}}+$ $\mathcal{N}_{\frac{3 \pi}{2}-\frac{\pi}{7}, \frac{\pi}{24}}$ (plus normalization).

Test Case 3: "Binary" This testcase was inspired by applications where the target distribution requires the values of the density to be "pixelized",. We alternate density values of 2 and 1 within the interval [19 $/ 16,29 \pi / 16]$ with the step $\pi / 16$, and a background noise $(1 . e-10)$, then normalize.

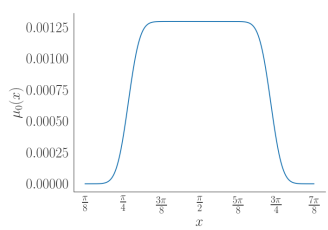

(a) Source Distribution $\mu_{0}$

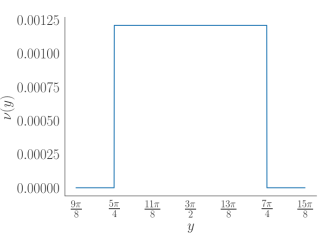

(b) Test Case 1: Uniform

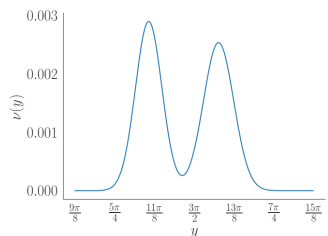

(c) Test Case 2: Two Gaussians

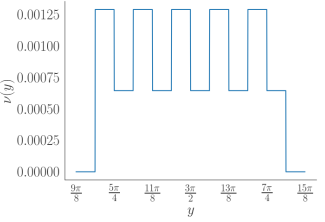

(d) Test Case 3: Binary

Figure 7: $\mu_{0}$ and Different desired Target densities.

Figure 8 compares the results obtained using the different optimization methods and backward ray tracing. The left column (approximated point source target) is the "optimal" $\nu_{0}$ and the center column is the resulting target obtained by ray tracing (binned) on the corresponding reflector. The discontinuous targets $\nu$ (test cases (A) and (C)) are clearly not in the range of the forward operator $\mathcal{F}$. The point source parameterization of the reflector performs a regularization through the already mentioned nonlinear convolution. The optimal solution still makes use of Diracs/parabola near the discontinuities as it provides the strongest slopes. Gold's method fails except for the smooth case (B), it is very sensitive to small density values.

In figure 9, we explore the choice of the raytracing method (section 2.4) with Adam optimization. Parameters have been tuned to use the same number of rays in both cases. It seems not to impact the optimization and justifies a preference for the more computationally efficient backward ray tracing. 

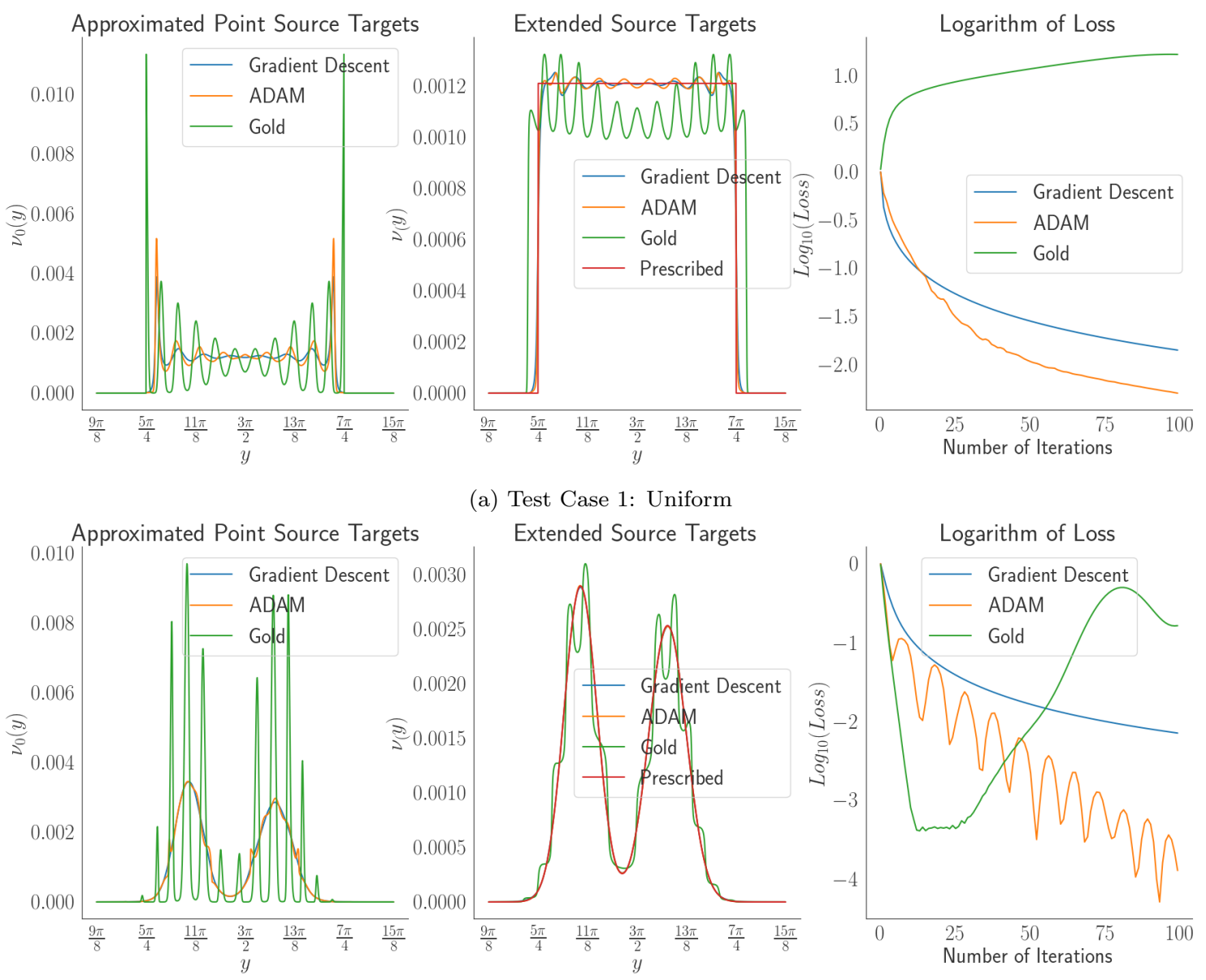

(a) Test Case 1: Uniform Extended Source Targets

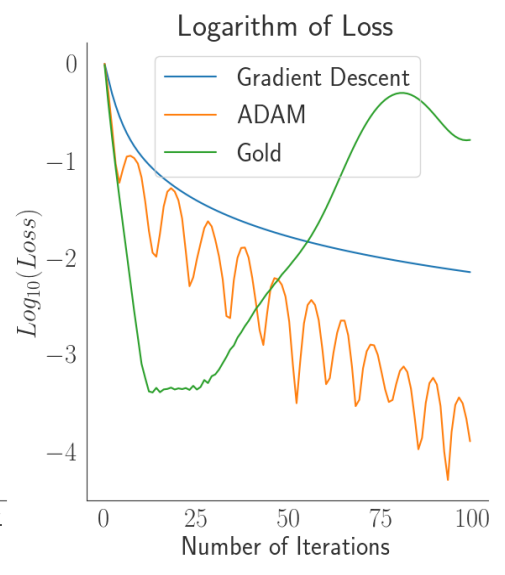

(b) Test Case 2: Two Gaussians
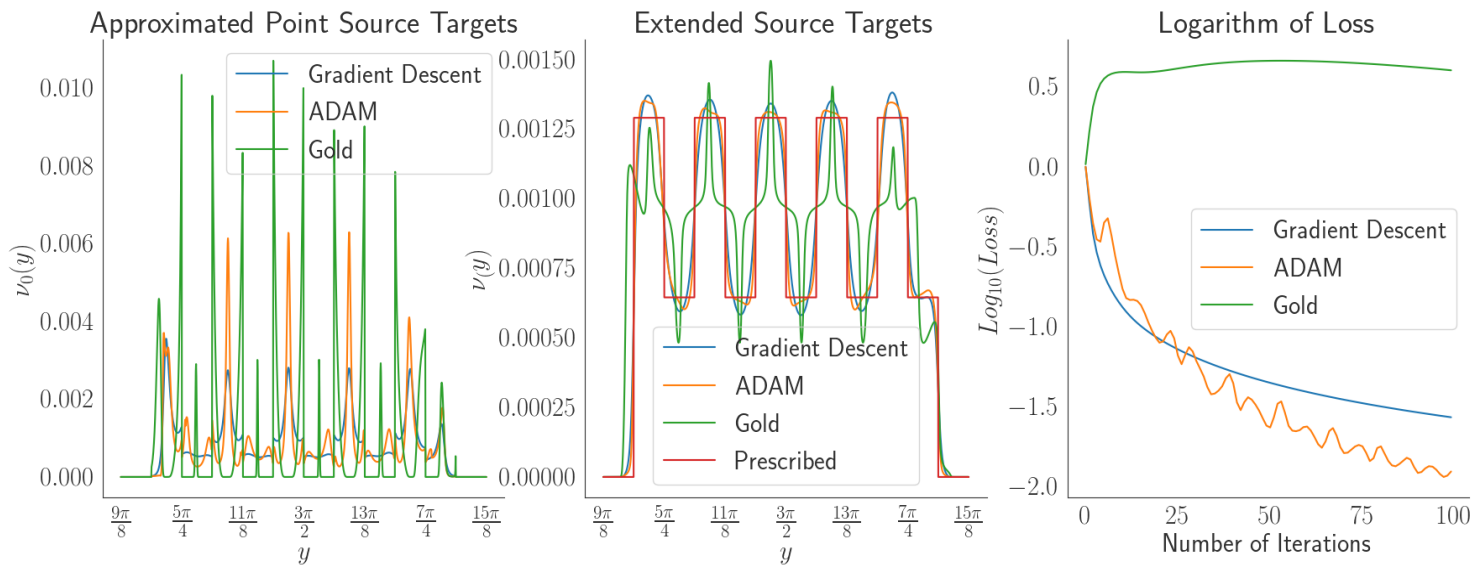

(c) Test Case 3: Binary

Figure 8: Comparison of different optimization methods. 

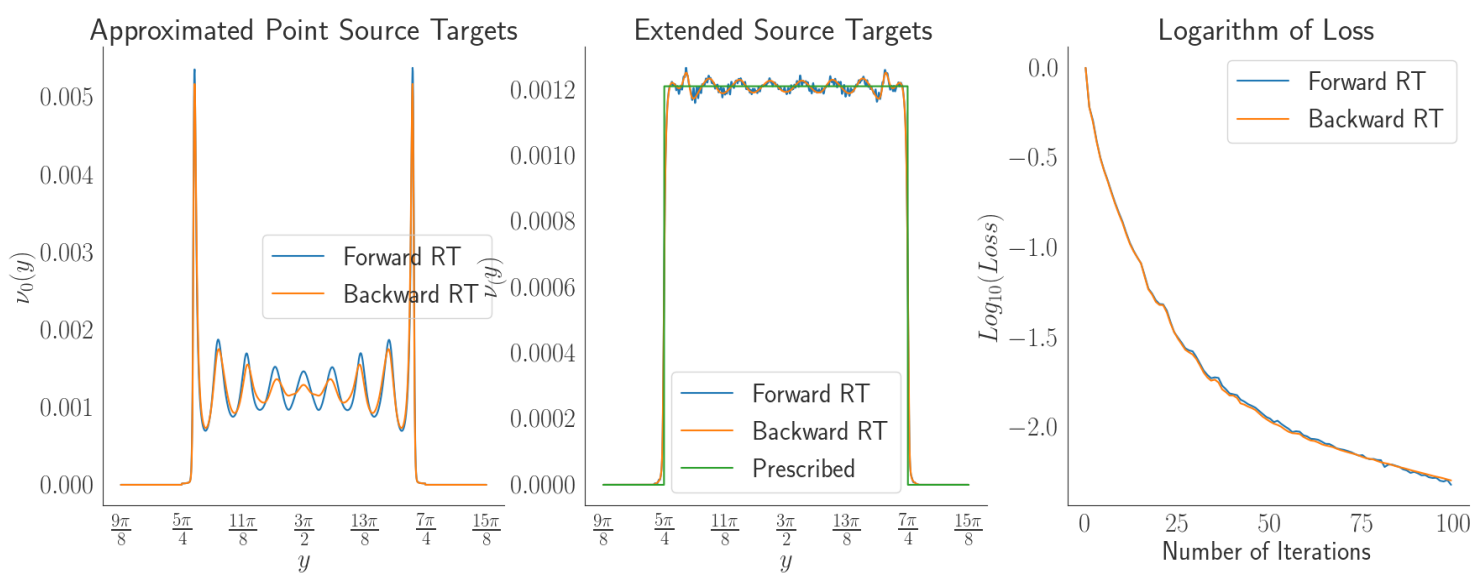

(a) Test Case 1: Uniform

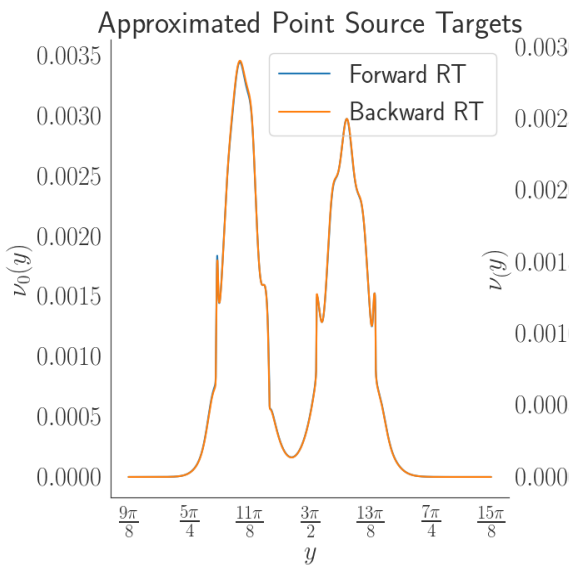

Extended Source Targets

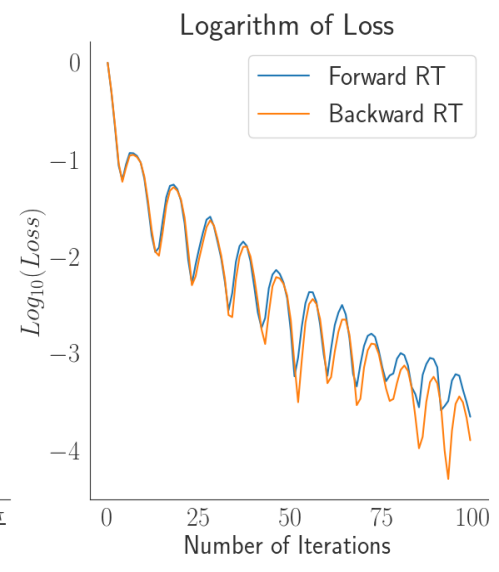

(b) Test Case 2: Two Gaussians

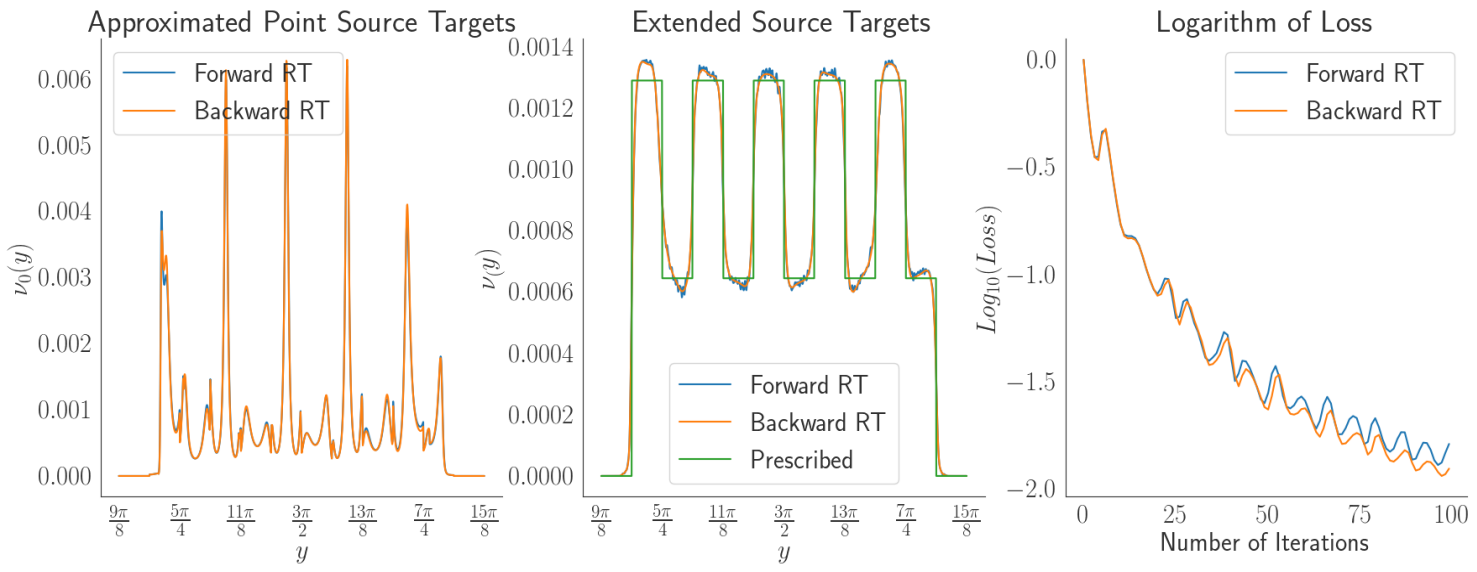

(c) Test Case 3: Binary

Figure 9: Comparison of forward/backward ray tracing. 


\section{Conclusion}

The proposed Optimal Transportation parameterization of the reflector offers theoretical guarantees for the optimization of a suitable Loss function at least for smooth data and provides a regularized solution. Our 1-D preliminary numerical study shows the approach is robust and converges at least to a local minimum. This research can be pursued in many directions:

- On the numerical side : the extension to $d=3$ (2D areflectors) of the code, testing Semi-Discrete Optimal Transportation solvers instead of the entropic solvers would also be relevant.

- A machine learning approach to parameterize the map $\mathcal{F}^{-1}: \nu \mapsto \nu_{0}$ with a convolutional neural network.

- Change of the optical setup, in the sense of changing the geometrical reflection law into a different, possibly more realistic reflection models as long as they are computationally efficient and differentiable (at least in the computational "automatic differentiation" sense). We are thinking for example about analytical BRDF models used in computer graphics and industrial design to approximate the scattering effects of various materials.

- It is possible to apply a multi-scale optimization strategy based on restarting with initializations obtained from a decreasing sequence of heights $R$. We did not report on this here as it did not make a significant difference in $1 \mathrm{D}$ but it may be useful in $2 \mathrm{D}$.

\section{Acknowledgements}

This work was supported by ROMSOC EID H2020 network under the Marie Curie Grant Agreement No. 765374.

\section{References}

[1] X.-J. Wang, On the design of a reflector antenna ii, Calculus of Variations and Partial Differential Equations 20 (3) (2004) 329-341. doi:10.1007/s00526-003-0239-4.

URL https://doi.org/10.1007/s00526-003-0239-4 
[2] F. Santambrogio, Optimal Transport for Applied Mathematicians: Calculus of Variations, PDEs, and Modeling, Progress in Nonlinear Differential Equations and Their Applications, Springer International Publishing, 2015.

URL https://books.google.fr/books?id=UOHHCgAAQBAJ

[3] S. A. Kochengin, V. I. Oliker, Computational algorithms for constructing reflectors, Computing and Visualization in Science 6 (1) (2003) 1521. doi:10.1007/s00791-003-0103-2.

URL https://doi .org/10.1007/s00791-003-0103-2

[4] G. Loeper, Regularity of optimal maps on the sphere: the quadratic cost and the reflector antenna, Archive for Rational Mechanics and Analysis 199 (1) (2011) 269-289. doi:10.1007/s00205-010-0330-x.

URL https://doi.org/10.1007/s00205-010-0330-x

[5] X.-J. Wang, On the design of a reflector antenna, Inverse Problems 12 (3) (1996) 351-375. doi:10.1088/0266-5611/12/3/013.

URL https://doi.org/10.1088/0266-5611/12/3/013

[6] M. Benning, M. Burger, Modern regularization methods for inverse problems, Acta Numerica 27 (2018) 1-111. doi:10.1017/S0962492918000016.

[7] M. Brand, D. A. Birch, Freeform irradiance tailoring for light fields, Opt. Express 27 (12) (2019) A611-A619. doi:10.1364/OE.27.00A611.

URL http: //www . opticsexpress . org/abstract . cfm?URI=oe-27-12-A611

[8] E. V. Byzov, S. V. Kravchenko, M. A. Moiseev, E. A. Bezus, L. L. Doskolovich, Optimization method for designing double-surface refractive optical elements for an extended light source, Opt. Express 28 (17) (2020) 24431-24443. doi:10.1364/OE.400609.

URL http: //www . opticsexpress . org/abstract . cfm?URI=oe-28-17-24431

[9] F. R. Fournier, W. J. Cassarly, J. P. Rolland, Fast freeform reflector generation using source-target maps, Opt. Express 18 (5) (2010) 5295-5304. doi:10.1364/OE.18.005295.

URL http: //www . opticsexpress . org/abstract . cfm?URI=oe-18-5-5295

[10] S. Wei, Z. Zhu, W. Li, D. Ma, Compact freeform illumination optics design by deblurring the response of extended sources, Opt. Lett. 46 (11) 
(2021) 2770-2773. doi:10.1364/OL.425075.

URL http://ol.osa.org/abstract.cfm?URI=ol-46-11-2770

[11] Y. Luo, Z. Feng, Y. Han, H. Li, Design of compact and smooth free-form optical system with uniform illuminance for led source, Opt. Express 18 (9) (2010) 9055-9063. doi:10.1364/OE.18.009055.

URL http: //www . opticsexpress . org/abstract . cfm?URI=oe-18-9-9055

[12] J.-D. Benamou, W. L. Ijzerman, G. Rukhaia, An Entropic Optimal Transport Numerical Approach to the Reflector Problem, working paper or preprint (Apr. 2020). doi:10.5281/zenodo.3755363.

URL https://doi.org/10.5281/zenodo. 3755363

[13] G. Peyré, M. Cuturi, Computational Optimal Transport, ArXiv e-prints (Mar. 2018). arXiv:1803.00567.

[14] K. Brix, Y. Hafizogullari, A. Platen, Designing illumination lenses and mirrors by the numerical solution of monge-ampère equations, Journal of the Optical Society of America A 32 (11) (2015) 2227. doi:10.1364/josaa.32.002227.

URL http://dx.doi.org/10.1364/JOSAA.32.002227

[15] P. Machado Manhães De Castro, Q. Mérigot, B. Thibert, Far-field reflector problem and intersection of paraboloids, Numerische Mathematik 134 (2) (2016) 389-411. doi:10.1007/s00211-015-0780-z.

URL https://hal.archives-ouvertes.fr/hal-00952720

[16] L. Romijn, J. ten Thije Boonkkamp, W. IJzerman, Freeform lens design for a point source and far-field target, Journal of the Optical Society of America A, Optics, Image Science and Vision 36 (11) (2019) 1926-1939. doi:10.1364/JOSAA.36.001926.

[17] L. Romijn, J. ten Thije Boonkkamp, W. IJzerman, Inverse reflector design for a point source and far-field target, Journal of Computational Physics 408 (5 2020). doi:10.1016/j.jcp.2020.109283.

[18] R. J. Berman, The sinkhorn algorithm, parabolic optimal transport and geometric monge-ampère equations, Numerische Mathematik 145 (4) (2020) 771-836. doi:10.1007/s00211-020-01127-x.

URL https://doi.org/10.1007/s00211-020-01127-x 
[19] J. Feydy, T. Séjourné, F.-X. Vialard, S.-i. Amari, A. Trouve, G. Peyré, Interpolating between optimal transport and mmd using sinkhorn divergences, in: K. Chaudhuri, M. Sugiyama (Eds.), Proceedings of the Twenty-Second International Conference on Artificial Intelligence and Statistics, Vol. 89 of Proceedings of Machine Learning Research, PMLR, 2019, pp. 2681-2690.

URL https://proceedings.mlr.press/v89/feydy19a.html

[20] L. Chizat, P. Roussillon, F. Léger, F.-X. Vialard, G. Peyré, Faster wasserstein distance estimation with the sinkhorn divergence, in: H. Larochelle, M. Ranzato, R. Hadsell, M. F. Balcan, H. Lin (Eds.), Advances in Neural Information Processing Systems, Vol. 33, Curran Associates, Inc., 2020, pp. 2257-2269.

URL https://proceedings.neurips.cc/paper/2020/file/ 17f98ddf040204eda0af36a108cbdea4-Paper.pdf

[21] J. Feydy, GEOMLOSS https://www.kernel-operations.io/geomloss/, 2019.

URL https://www.kernel-operations.io/geomloss/

[22] G. Luise, A. Rudi, M. Pontil, C. Ciliberto, Differential properties of sinkhorn approximation for learning with wasserstein distance, in: S. Bengio, H. Wallach, H. Larochelle, K. Grauman, N. Cesa-Bianchi, R. Garnett (Eds.), Advances in Neural Information Processing Systems, Vol. 31, Curran Associates, Inc., 2018.

URL https://proceedings.neurips.cc/paper/2018/file/ 3fc2c60b5782f641f76bcefc39fb2392-Paper.pdf

[23] G. Chazareix, Extended Source Reflector Problem Code https://github.com/NightWinkle/ExtendedSourceReflectorProblem, 2020 .

URL https://github.com/NightWinkle/ExtendedSourceReflectorProblem

[24] R. Gold, An iterative unfolding method for response matrices (12 1964). doi:10.2172/4634295.

URL https://www.osti.gov/biblio/4634295

[25] D. P. Kingma, J. Ba, Adam: A method for stochastic optimization (2017). arXiv:1412.6980. 
[26] C. Villani, Optimal Transport: Old and New, Grundlehren der mathematischen Wissenschaften, Springer Berlin Heidelberg, 2008.

URL https://books.google.fr/books?id=NZXiNAEACAAJ

\section{Annex}

Proposition 1 (On the Point source Optimal Transportation reflector)

(i) $\mathcal{R}_{f_{0}}, f_{0}$ solution of (6) is a strictly convex curve (surface for $d=3$ ).

(ii) If $\mu_{0}$ and $\nu_{0}$ are Hoelder continuous and positive, $Y$ c-convex then the Ma-Trudinger-Wang [4] [5] regularity theory applies, $f_{0}$ is bounded in $C^{2, \alpha},(0<\alpha<1)$.

(iii) $\tan \left(n_{0}\left(x_{0}\right)\right)=\partial_{x_{0}} f_{0}\left(x_{0}\right)$, in particular the strict monotony of $x_{0} \rightarrow$ $n_{0}\left(x_{0}\right)$ carries over to $x_{0} \rightarrow \partial_{x_{0}} f_{0}\left(x_{0}\right)$.

\section{Proof 3.}

(i) This is from [1] Lemma 3.2: we know that

$$
\mathcal{R}_{f_{0}}=\left\{\hat{x}_{0}\left(\inf _{y \in Y} P_{y}\left(x_{0}\right)\right), x_{0} \in X_{0}\right\},
$$

where

$$
P_{y}\left(x_{0}\right):=\frac{e^{-g_{0}(y)}}{1-\cos \left(y-x_{0}\right)} .
$$

The family $\left\{x_{0} \mapsto \hat{x}_{0} P_{y}\left(x_{0}\right)\right\}_{y \in Y}$ is composed of parabolae with focal at $O_{0}$ and axis in the direction $y$. The Optimal Transportation reflector $\mathcal{R}_{f_{0}}$ is the envelope of these parabolae. It is continuous and strictly convex.

(iii)

$$
\begin{aligned}
\partial_{x_{0}} f_{0}\left(x_{0}\right)= & \left.\partial_{x_{0}} c\left(x_{0}, y_{x_{0}}\right), \text { (from }(9)\right) \\
& =-\frac{\sin \left(y_{x_{0}}-x_{0}\right)}{1-\cos \left(y_{x_{0}}-x_{0}\right)} \\
& =-\cot \left(\frac{1}{2}\left(y_{x_{0}}-x_{0}\right)\right) \\
& =\tan \left(n_{0}\left(x_{0}\right)\right) \\
& \left(n_{0}\left(x_{0}\right)=\frac{1}{2}\left(y_{x_{0}}-x_{0}\right)-\frac{\pi}{2} \text { with our angle notations }\right) .
\end{aligned}
$$


Proposition 2. (Re-parameterization of $\mathcal{R}_{f_{0}}$ )

Let us assume that $X_{0}=X_{s}=\mathbb{S}_{+}^{1}$ for all $s$ and $\eta<\frac{R}{2}$. Then, for all $s \in S$ there exists and a function $f_{s}: X_{s} \rightarrow \mathbb{R}^{+}$such that:

(i) The following re-parameterization of the reflector holds:

$$
\mathcal{R}_{f_{0}}=\mathcal{R}_{f_{s}}:=\left\{\hat{x}_{s} e^{f_{s}\left(x_{s}\right)}, x_{s} \in X_{s}\right\} .
$$

where $\hat{x}_{s}$ is the unit vector in the direction $x_{s}$ from $O_{s}$.

(ii) The inner normal angle in the (39) parameterization, denoted $n_{s}\left(x_{s}\right)$ is given by

$$
\tan \left(n_{s}\left(x_{s}\right)\right)=\frac{\partial_{x_{s}} f_{s}\left(x_{s}\right) \cos \left(x_{s}\right)+\sin \left(x_{s}\right)}{\cos \left(x_{s}\right)-\partial_{x_{s}} f_{s}\left(x_{s}\right) \sin \left(x_{s}\right)} .
$$

(iii) The map $f_{0} \mapsto f_{s}$ is continuous for the $\mathcal{C}^{1}\left(\mathbb{S}_{-}^{1}\right)$ topology.

\section{Proof 4.}

A preliminary is to verify that the source patch $S$ remains strictly inside the reflector convex envelope. Based on the envelope of parabolae property (proposition 1 (i)) the abcissa of the intersection of the reflector with the axis supporting the patch (orthogonal to $\frac{\hat{\pi}}{2}$ ) is bounded below by the abcissa of the intersection of the axis with the parabola: $x_{0} \in \mathbb{S}_{-}^{1} \mapsto \frac{C}{1-\cos \left(\pi-x_{0}\right)}$ and symmetrically $x_{0} \in \mathbb{S}_{-}^{1} \mapsto \frac{C}{1-\cos \left(x_{0}\right)}$ which is reached respectively for $x_{0}=0$ and $x_{0}=\pi$. The constant is fixed by $\frac{C}{1-\cos \left(\frac{3 \pi}{2}\right)}=R$ as in (1) and therefore the intersection is bounded below by $\frac{R}{2}$, hence the condition $\eta<\frac{R}{2}$.

(i) All points $O_{s}=(0, s)$ on $S$ are therefore in the convex envelope of $\mathcal{R}_{f_{0}}$. The smoothness and strict convexity of the reflector therefore guarantee that any point $(s, 0)$ can be connected to any point on the reflector without intersecting the reflector anywhere else. This provides the re-parameterization (39) and also the uniqueness of $x_{s} \mapsto f_{s}\left(x_{s}\right)$.

(ii) is a direct consequence of the parameterization (39). 
(iii) Define $\mathcal{A}_{s}\left(x_{0}\right)$ as the angle of the vector connecting the shifted source $O_{s}$ to the point $\mathcal{R}_{f_{0}}\left(x_{0}\right):=\hat{x}_{0} e^{f_{0}\left(x_{0}\right)}$. Using parameterization (39) one has

$$
\mathcal{A}_{s}\left(x_{0}\right):=\arccos \left(\frac{\cos \left(x_{0}\right) e^{f_{0}\left(x_{0}\right)}-s}{\sqrt{e^{2 f_{0}\left(x_{0}\right)}-2 s e^{f_{0}\left(x_{0}\right)} \cos \left(x_{0}\right)+s^{2}}}\right),
$$

and

$$
f_{s}\left(\mathcal{A}_{s}\left(x_{0}\right)\right):=\log \left(\sqrt{e^{2 f_{0}\left(x_{0}\right)}-2 s e^{f_{0}\left(x_{0}\right)} \cos \left(x_{0}\right)+s^{2}}\right) .
$$

The map $x_{0} \mapsto \mathcal{A}_{s}\left(x_{0}\right)$ is bijective, smooth and differentiable like $f_{0}$ ( a consequence of proposition 1 (i)-(ii)). By construction at all points on the reflector $n_{0}\left(x_{0}\right)=n_{s}\left(A_{s}\left(x_{0}\right)\right)$ where $n_{0}$ and $n_{s}$ are defined respectively in proposition 1 (iii) and proposition 2 (ii). Taking the derivative in $x_{0}$ we get

$$
\partial_{x_{0}} A_{s} \partial_{x_{0}} n_{s}\left(A_{s}\right)=\partial_{x_{0}} n_{0},
$$

and using the strict convexity of the reflector (proposition 1 (iii)), we find that $\partial_{x_{0}} A_{s}$ cannot vanish. Applying the inverse function theorem $\mathcal{A}_{s}$ is therefore a diffeormorphism from $S_{+}^{1}$ onto itself. We can now re-write $f_{s}$ using the new parameterization $x_{s}=\mathcal{A}_{s}\left(x_{0}\right)$ :

$$
f_{s}\left(x_{s}\right)=\log \left(\sqrt{e^{2 f_{0}\left(\mathcal{A}_{s}^{-1}\left(x_{s}\right)\right)}-2 s \cos \left(\mathcal{A}_{s}^{-1}\left(x_{s}\right)\right) e^{f_{0}\left(\mathcal{A}_{s}^{-1}\left(x_{s}\right)\right)}+s^{2}}\right),
$$

which is continuously differentiable with derivative:

$$
\begin{gathered}
\partial_{x_{s}} f_{s}\left(x_{s}\right)=\frac{e^{f_{0}\left(\mathcal{A}_{s}^{-1}\left(x_{s}\right)\right)}\left(\partial_{x_{s}} \mathcal{A}_{s}^{-1}\right)\left(x_{s}\right)}{e^{2 f_{0}\left(\mathcal{A}_{s}^{-1}\left(x_{s}\right)\right)}-2 s \cos \left(\mathcal{A}_{s}^{-1}\left(x_{s}\right)\right) e^{f_{0}\left(\mathcal{A}_{s}^{-1}\left(x_{s}\right)\right)}+s^{2}} Q \\
Q:=e^{f_{0}\left(\mathcal{A}_{s}^{-1}\left(x_{s}\right)\right)} \partial_{x_{0}} f_{0}\left(\mathcal{A}_{s}^{-1}\left(x_{s}\right)\right)+s \sin \left(\mathcal{A}_{s}^{-1}\left(x_{s}\right)\right)-s \cos \left(\mathcal{A}_{s}^{-1}\left(x_{s}\right)\right) \partial_{x_{0}} f_{0}\left(\mathcal{A}_{s}^{-1}\left(x_{s}\right)\right) .
\end{gathered}
$$

Note that $f_{s}$ and $\partial_{x_{s}} f_{s}\left(x_{s}\right)$ are expressed analytically using $f_{0}, \partial_{x_{0}} f$, $\mathcal{A}_{s}^{-1}$ and $\partial_{x_{s}} \mathcal{A}_{s}^{-1}$ (As fractions with non-vanishing denominators and bounded numerators). Thereof, in order to demonstrate $C^{1}$ convergence, all we need to do is to establish the continuous dependency of $\mathcal{A}_{s}^{-1}$ and $\partial_{x_{s}} \mathcal{A}_{s}^{-1}$ on $f_{0}$. This is just a consequence of the inverse function theorem: As $\partial_{x_{0}} \mathcal{A}_{s}$ depends continuously on $f_{0}$ and $\partial_{x_{s}} \mathcal{A}_{s}^{-1}$ can be expressed by $1 / \partial_{x_{0}} \mathcal{A}_{s}$.

The continuity of $f_{0} \mapsto f_{s}$ for the $C^{1}$ topology follows. 
Theorem 1. (Continuity of $\mathcal{F}$ ) Under the assumptions of proposition (1)-(2), $\mathcal{F}$ is continuous for the weak convergence in $\mathcal{P}\left(S_{+}^{1}\right)$.

Proof 5. Let $\left\{\nu_{0, k}\right\}_{k \in \mathbb{N}}$ be a weakly convergent sequence converging to $\nu_{0}$. We need to verify that $\mathcal{F}\left(\nu_{0, k}\right)$ also converges weakly to $\mathcal{F}\left(\nu_{0}\right)$. Note that (see remark 2) $\mathcal{F}(\nu)$ can be expressed as $\int_{S} T_{s}^{\nu} \# \mu_{0}$. By the linearity of the integration, all we need is to verify that $T_{s}^{\nu_{0}, k} \# \mu_{0}$ converge weakly to $T_{s}^{\nu_{0}} \# \mu_{0}$ for all $s$.

The stability of $\nu_{0} \mapsto T_{0}^{\nu_{0}}$ is a classical result (see [26] corollary 5.23), where the convergence of sequence $\left(T_{0}^{\nu_{0, k}}\right)$ (built from (9) using the sequence of $\left.\left(f_{0, k}\right)\right)$ holds in probability:

$$
\forall \epsilon>0 \quad \mu_{0}\left[\left\{x \in \mathbb{S}_{+} \mid d\left(T_{0}^{\nu_{0}}(x), T_{0}^{\nu_{0, k}}(x)\right)>\epsilon\right\}\right] \stackrel{k \rightarrow \infty}{\longrightarrow} 0 .
$$

Note that under the assumptions, $T_{0}^{\nu}$ is induced by the reflection from the reflector with the continuous normal $n=n^{\nu}$. Hence the convergence of $T_{0}^{\nu_{0, k}}$ translates into the convergence of $n^{\nu_{0, k}}$. Since we have the $C^{1}$ continuity of $f_{0}$ to $f_{s}$, and since for all $s, T_{s}^{\nu_{0, k}}$ is just another reflection using the reparametrized normal $n_{s}^{\nu_{0, k}}$, the above convergence in probability holds also for all $T_{s}^{\nu_{0 . k}}$.

Now all we need is to verify that for the sequence of maps $T^{k}: \mathbb{S}_{+} \rightarrow \mathbb{S}_{-}$, converging in probability to the map $T$ with respect to the measure $\mu$, the pushforward measures $T^{k} \# \mu_{0}$ converge weakly to $T \# \mu_{0}$. For this we use the Portmanteau theorem to check the following convergence for all bounded Lipschitz functions $\phi$ :

$$
\int_{\mathbb{S}_{-}} \phi(y) T^{k} \# \mu_{0}(y) \stackrel{k \rightarrow \infty}{\longrightarrow} \int_{S_{-}} \phi(y) T \# \mu_{0}(y) .
$$

Fix the function $\phi$ with a Lipschitz constant $L_{\phi}$ and an $\epsilon>0$. Using the change of variable formula, Lipschitz property of $\phi$ and boundedness of $\mathbb{S}$ we get: 


$$
\begin{aligned}
& \left|\int_{\mathbb{S}_{-}} \phi(y) T^{k} \# \mu_{0}(y)-\int_{S_{-}} \phi(y) T \# \mu_{0}(y)\right| \leq \\
& \int_{\mathbb{S}_{+}}\left|\phi\left(T^{k}(x)\right)-\phi(T(x))\right| \mu_{0}(x) \leq \\
& \int_{\mathbb{S}_{+}} L_{\phi}\left|d_{\mathbb{S}}\left(T^{k}(x), T(x)\right)\right| \mu_{0}(x) \leq \\
& \int_{\mathbb{S}_{+} \backslash B_{\epsilon}} \epsilon L_{\phi} \mu_{0}(x)+\int_{B_{\epsilon}} \operatorname{diam}\left(\mathbb{S}_{+}\right) L_{\phi} \mu_{0}(x) \leq \\
& \epsilon L_{\phi}+\operatorname{diam}\left(S_{+}\right) L_{\phi} \mu_{0}\left[B_{\epsilon}\right] .
\end{aligned}
$$

Where $B_{\epsilon}$ is the set:

$$
B_{\epsilon}:=\left\{x \in \mathbb{S}_{+} \mid d\left(T_{0}^{\nu_{0}}(x), T_{0}^{\nu_{0, k}}(x)\right)>\epsilon\right\} .
$$

Due to the convergence in probability of $T^{k}$, the quantity $\mu_{0}\left[B_{\epsilon}\right] \stackrel{k \rightarrow \infty}{\longrightarrow} 0$, which concludes the proof. 